\title{
Molecular Structure and Vibrational Spectra of 2-Ethylhexyl Acrylate by Density Functional Theory Calculations
}

\author{
Ottman Belaidi, ${ }^{1}$ Tewfik Bouchaour, ${ }^{1}$ and Ulrich Maschke ${ }^{2}$ \\ ${ }^{1}$ Laboratoire de Recherche sur les Macromolécules, Faculté des Sciences, Université Abou Bekr Belkaid, BP 119-13000, Tlemcen, Algeria \\ ${ }^{2}$ UMET (Unité Matériaux et Transformations), UMR CNRS No. 8207, Université Lille 1-Sciences et Technologies, \\ 59655 Villeneuve d'Ascq Cedex, France
}

Correspondence should be addressed to Ottman Belaidi; othmanblaidi@gmail.com

Received 26 February 2013; Accepted 28 April 2013

Academic Editor: Dipakranjan Mal

Copyright (C) 2013 Ottman Belaidi et al. This is an open access article distributed under the Creative Commons Attribution License, which permits unrestricted use, distribution, and reproduction in any medium, provided the original work is properly cited.

The Fourier transform infrared spectra (FTIR) of 2-ethylhexyl acrylate have been measured in liquid phase. The molecular geometry, vibrational frequencies, and infrared intensities have been calculated by using density functional theory. We found two local minima representing s-cis and s-trans conformations for 2-ethylhexyl acrylate molecule. The optimized geometries at DFT//B3LYP/6-311+G** are in good agreement with electron diffraction data of methyl acrylate for the acrylic group. The two conformers were used for the interpretation of the experimental infrared spectrum of title compound. PED calculations are represented for a more complete and concise assignment. There is one band in the infrared spectrum at $1646 \mathrm{~cm}^{-1}$ that definitely indicates the conformer with s-trans arrangement of acrylic moiety to be present or not in the liquid 2-ethylhexyl acrylate.

\section{Introduction}

Alkyl acrylates are important monomers used in the manufacture of polymers and copolymers. Acrylates give polymers exhibiting outstanding transparency and aging proprieties which have made them of interest in a wide variety of applications [1-4]. The structural data and the preferred molecular conformation of these acrylates molecules would be important for basic understanding of these polymers [5].

2-Ethylhexyl acrylate is used for production of paint, adhesive, and paper coating trade. This monomer imparts flexibility and water resistance to the polymers $[1,6]$. It is also used for curing polymeric materials [7-15].

To our best knowledge no structural data or detailed interpretation of the vibrational spectra of 2-ethylhexyl acrylate is presented in the literature. This prompted us to look into the vibrational spectroscopy of 2-ethylhexyl acrylate more carefully.

Many reports, experimental [16-21] and theoretical [20, 22 ], show that acrylates and related compounds exhibit rotational isomerism with the planar s-trans and s-cis heavyatom structures being the energetically most stable conformations. However, uncertainty continues to exist regarding the relative stability of the two conformers. Gas electron diffraction studies on methyl methacrylate [20] suggested the cis/trans ratios to be equal to $2: 1$. On the other hand, the IR spectrum of methyl methacrylate in Ar low temperature matrix suggested an inverse ratio [23]. Many experimental and theoretical studies on methyl acrylate reported that the $\mathrm{s}$-cis conformer is more stable than the s-trans conformer [24-29]. However, Bowles et al. [30] assumed that the s-trans conformer is more stable.

\section{Experimental}

2-Ethylhexyl acrylate (purity greater than 99\%) was purchased from Sigma-Aldrich and it was used as it is without further purification. The infrared spectra of liquid films placed between the KBR windows were recorded within $4000-700 \mathrm{~cm}^{-1}$ range with a Perkin Elmer FTIR System-2000 model.

\section{Computational}

The optimizations of the stable conformers were conducted with the density functional theory using $6-311+\mathrm{G}^{* *}$ basis 

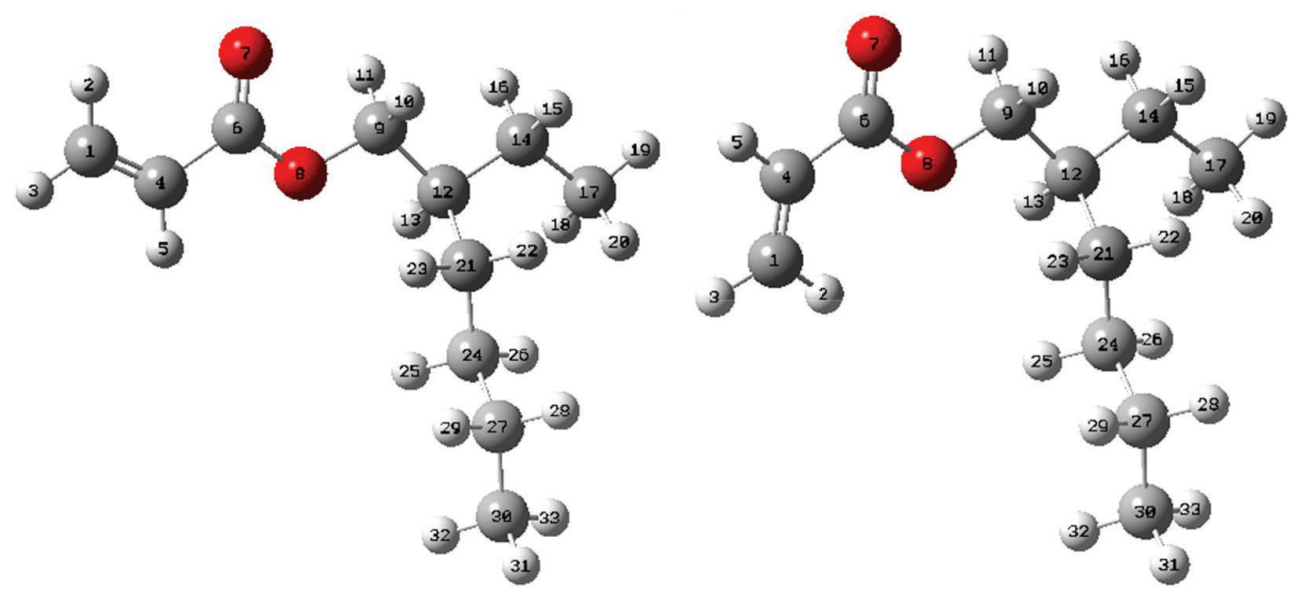

FIGURE 1: 2-Ethylhexyl acrylate molecule s-cis and s-trans conformations.

sets. The DFT calculations were performed using Becke's 3parameter (local, nonlocal, Hartree-Fock) hybrid exchange functionals with Lee-Yang-Parr correlational functionals (B3LYP) [31, 32].

The harmonic vibrational frequencies of the stable conformations were calculated at the same level of theories used for the calculated optimized geometries. The calculated frequencies were scaled down by the wavenumber linear scaling procedure (WLS) of Yoshida et al. $[33,34]$ using the following equation:

$$
\frac{v_{\text {obs }}}{v_{\text {calc }}}=1.0087-0.0000163 v_{\text {calc }} .
$$

All the calculations were carried out with the Gaussian 03 program [35]. The vibrational assignments of the normal modes were provided on the basis of the calculated PEDs by using the program GAR2PED [36].

\section{Geometry Optimizations and Energies}

The geometries optimizations were conducted at the B3LYP/ $6-311+\mathrm{G}^{* *}$ assuming the $\mathrm{s}$-cis and s-trans conformations. All the optimized geometries are recognized as true minima due to the lack of imaginary harmonic frequencies. The results are given in Table 1 for s-cis and s-trans conformers, respectively. For atoms numbering, see Figure 1.

In Table 1, the calculated bond lengths, bond angles, and dihedral angles have approximately the same values for the $\mathrm{s}$-cis and s-trans conformers. However, some changes occur in going from the $s$-cis to the s-trans conformation. The bond angles $\mathrm{C}_{1} \mathrm{C}_{4} \mathrm{C}_{6}$ and $\mathrm{C}_{4} \mathrm{C}_{6} \mathrm{O}_{8}$ increase by $3.8^{\circ}$ and $2.7^{\circ}$, respectively, while $\mathrm{C}_{4} \mathrm{C}_{6} \mathrm{O}_{7}$ decreases by $2.5^{\circ}$.

The $\mathrm{X}$ ray structural data of s-cis methyl acrylate reported by Egawa et al. [21] and ab initio structure of s-trans methyl acrylate reported by Tsuji et al. [20] are very close to those we found for 2-ethylhexyl acrylate molecule.

The zero-point corrected energy of the s-cis conformation is $365.102 \times 10^{6} \mathrm{cal} / \mathrm{mol}$ showing more stability than the calculated one for the s-trans conformation. The energy for the latter conformation is $365.101 \times 10^{6} \mathrm{cal} / \mathrm{mol}$.

\section{Vibrational Analysis}

The most stable conformers, s-cis and s-trans 2-ethylhexyl acrylate, belong to the $C_{1}$ point group. Each conformer represents 93 normal vibrational modes. We juxtaposed the experimental IR spectrum with the computed ones for both conformers and we checked whether we can recognize them or not. Tables 2 and 3 represent the calculated and scaled fundamental wavenumbers, intensities of vibrational peaks, and potential energy distribution along the internal coordinates obtained by DFT//B3LYP/6-311+G** level of theory. The corresponding experimental wavenumbers together with assignments are also reported in these tables. The potential energy distributions are given as per the internal coordinate system recommended by Pulay et al. using DFT [37].

5.1. The CH Stretching Vibrations Region (3200-2800 $\mathrm{cm}^{-1}$, Figure 2). In this region, the calculations reveal the existence of 20 vibrational normal modes for both the s-cis and strans conformers. These normal modes are of approximately the same energies and intensities for both conformers. In the IR spectrum in this region (Figure 2(a)) there are three groups of bands, two groups of strong bands and one group of a very weak band. In the latter there are three bands of very weak intensity at 3104,3069 , and $3038 \mathrm{~cm}^{-1}$ which are well reproduced by the theoretical bands 3093, 3047, and $3012 \mathrm{~cm}^{-1}$, respectively. These bands are ascribed to the $\mathrm{C}_{1} \mathrm{H}$ and $\mathrm{C}_{4} \mathrm{H}$ stretching modes of vibrations of the vinyl group. The second group is composed of the strongest peaks at 2961 and $2931 \mathrm{~cm}^{-1}$. These two bands are well reproduced by the theoretical bands 2963 and $2953 \mathrm{~cm}^{-1}$, respectively. The third group is composed of one peak slightly asymmetric at $2875 \mathrm{~cm}^{-1}$ in the experimental IR spectrum; this band is reproduced at $2903 \mathrm{~cm}^{-1}$ in the theoretical calculations. The remaining bands below $2900 \mathrm{~cm}^{-1}$ with higher intensity in these series of $\mathrm{CH}$ stretching vibrational modes may be assigned to reproduce the broad shoulder at $2861 \mathrm{~cm}^{-1}$. All the theoretical peaks located between 3000 and $2860 \mathrm{~cm}^{-1}$ are ascribed to $\mathrm{CH}$ stretching of the methylene groups in 
TABLE 1: Optimized geometries of s-cis and s-trans 2-ethylhexyl acrylate conformations by DFT//B3LYP/6-311+G ${ }^{* *}$.

\begin{tabular}{|c|c|c|c|c|}
\hline Internal coordinates & $\begin{array}{c}\text { Level of theory } \\
\text { DFT/6-311+G** } \\
\text { s-cis EHA }\end{array}$ & $\begin{array}{l}\text { s-cis methyl } \\
\text { acrylate }^{\mathrm{a}}\end{array}$ & $\begin{array}{c}\text { DFT/6-311+G }{ }^{* *} \\
\text { s-trans EHA }\end{array}$ & $\begin{array}{l}\text { s-trans methyl } \\
\text { acrylate }^{\mathrm{b}}\end{array}$ \\
\hline \multicolumn{5}{|l|}{ Bond lengths $(\AA ̊)$} \\
\hline $\mathrm{C}_{1} \mathrm{H}_{2}$ & 1.084 & & 1.083 & 1.074 \\
\hline $\mathrm{C}_{1} \mathrm{H}_{3}$ & 1.083 & & 1.084 & 1.075 \\
\hline $\mathrm{C}_{1} \mathrm{C}_{4}$ & 1.331 & $1.341(7)$ & 1.332 & 1.319 \\
\hline $\mathrm{C}_{4} \mathrm{H}_{5}$ & 1.084 & & 1.084 & 1.075 \\
\hline $\mathrm{C}_{4} \mathrm{C}_{6}$ & 1.486 & $1.484(6)$ & 1.484 & 1.484 \\
\hline $\mathrm{C}_{6} \mathrm{O}_{7}$ & 1.210 & $1.211(2)$ & 1.211 & 1.191 \\
\hline $\mathrm{C}_{6} \mathrm{O}_{8}$ & 1.351 & $1.349(3)$ & 1.348 & 1.325 \\
\hline $\mathrm{O}_{8} \mathrm{C}_{9}$ & 1.448 & $1.439(3)$ & 1.448 & 1.417 \\
\hline $\mathrm{C}_{9} \mathrm{H}_{10}$ & 1.093 & & 1.093 & \\
\hline $\mathrm{C}_{9} \mathrm{H}_{11}$ & 1.093 & & 1.092 & \\
\hline $\mathrm{C}_{9} \mathrm{C}_{12}$ & 1.526 & & 1.526 & \\
\hline $\mathrm{C}_{12} \mathrm{H}_{13}$ & 1.098 & & 1.098 & \\
\hline $\mathrm{C}_{12} \mathrm{C}_{14}$ & 1.543 & & 1.543 & \\
\hline $\mathrm{C}_{14} \mathrm{H}_{15}$ & 1.097 & & 1.097 & \\
\hline $\mathrm{C}_{14} \mathrm{H}_{16}$ & 1.096 & & 1.096 & \\
\hline $\mathrm{C}_{14} \mathrm{C}_{17}$ & 1.531 & & 1.531 & \\
\hline $\mathrm{C}_{17} \mathrm{C}_{18}$ & 1.094 & & 1.094 & \\
\hline $\mathrm{C}_{17} \mathrm{H}_{19}$ & 1.093 & & 1.093 & \\
\hline $\mathrm{C}_{17} \mathrm{H}_{20}$ & 1.092 & & 1.092 & \\
\hline $\mathrm{C}_{12} \mathrm{C}_{21}$ & 1.549 & & 1.549 & \\
\hline $\mathrm{C}_{21} \mathrm{H}_{22}$ & 1.097 & & 1.097 & \\
\hline $\mathrm{C}_{21} \mathrm{H}_{23}$ & 1.094 & & 1.094 & \\
\hline $\mathrm{C}_{21} \mathrm{C}_{24}$ & 1.534 & & 1.534 & \\
\hline $\mathrm{C}_{24} \mathrm{H}_{25}$ & 1.097 & & 1.097 & \\
\hline $\mathrm{C}_{24} \mathrm{H}_{26}$ & 1.095 & & 1.095 & \\
\hline $\mathrm{C}_{24} \mathrm{C}_{27}$ & 1.533 & & 1.533 & \\
\hline $\mathrm{C}_{27} \mathrm{H}_{28}$ & 1.097 & & 1.097 & \\
\hline $\mathrm{C}_{27} \mathrm{H}_{29}$ & 1.097 & & 1.096 & \\
\hline $\mathrm{C}_{27} \mathrm{C}_{30}$ & 1.531 & & 1.531 & \\
\hline $\mathrm{C}_{30} \mathrm{H}_{31}$ & 1.094 & & 1.093 & \\
\hline $\mathrm{C}_{30} \mathrm{H}_{32}$ & 1.094 & & 1.094 & \\
\hline $\mathrm{C}_{30} \mathrm{H}_{33}$ & 1.094 & & 1.094 & \\
\hline \multicolumn{5}{|l|}{ Bond angles $\left({ }^{\circ}\right)$} \\
\hline $\mathrm{H}_{2} \mathrm{C}_{1} \mathrm{H}_{3}$ & 118.049 & & 117.591 & \\
\hline $\mathrm{H}_{2} \mathrm{C}_{1} \mathrm{C}_{4}$ & 120.479 & & 121.432 & 121.7 \\
\hline $\mathrm{C}_{1} \mathrm{C}_{4} \mathrm{H}_{5}$ & 122.220 & & 121.739 & 122.1 \\
\hline $\mathrm{C}_{1} \mathrm{C}_{4} \mathrm{C}_{6}$ & 121.072 & $120.3(8)$ & 124.970 & 124.2 \\
\hline $\mathrm{C}_{4} \mathrm{C}_{6} \mathrm{O}_{7}$ & 125.710 & $126.1(5)$ & 123.066 & 122.9 \\
\hline $\mathrm{C}_{4} \mathrm{C}_{6} \mathrm{O}_{8}$ & 110.564 & $110.3(3)$ & 113.320 & 113.7 \\
\hline $\mathrm{C}_{6} \mathrm{O}_{8} \mathrm{C}_{9}$ & 116.570 & $116.4(5)$ & 116.493 & 116.8 \\
\hline $\mathrm{O}_{8} \mathrm{C}_{9} \mathrm{H}_{10}$ & 108.658 & & 108.697 & \\
\hline $\mathrm{O}_{8} \mathrm{C}_{9} \mathrm{H}_{11}$ & 108.313 & & 108.339 & \\
\hline $\mathrm{O}_{8} \mathrm{C}_{9} \mathrm{C}_{12}$ & 108.522 & & 108.488 & \\
\hline
\end{tabular}


TABLe 1: Continued.

\begin{tabular}{|c|c|c|c|c|}
\hline Internal coordinates & $\begin{array}{c}\text { Level of theory } \\
\text { DFT/6-311+G* } \\
\text { s-cis EHA }\end{array}$ & $\begin{array}{l}\text { s-cis methyl } \\
\text { acrylate }^{\mathrm{a}}\end{array}$ & $\begin{array}{c}\text { DFT/6-311+G }{ }^{* *} \\
\text { s-trans EHA }\end{array}$ & $\begin{array}{c}\text { s-trans methyl } \\
\text { acrylate }^{\mathrm{b}}\end{array}$ \\
\hline $\mathrm{C}_{9} \mathrm{C}_{12} \mathrm{C}_{14}$ & 108.099 & & 108.115 & \\
\hline $\mathrm{C}_{12} \mathrm{C}_{14} \mathrm{H}_{15}$ & 108.729 & & 108.720 & \\
\hline $\mathrm{C}_{12} \mathrm{C}_{14} \mathrm{H}_{16}$ & 108.539 & & 108.544 & \\
\hline $\mathrm{C}_{12} \mathrm{C}_{14} \mathrm{C}_{17}$ & 115.410 & & 115.400 & \\
\hline $\mathrm{C}_{14} \mathrm{C}_{17} \mathrm{H}_{18}$ & 111.304 & & 111.345 & \\
\hline $\mathrm{C}_{14} \mathrm{C}_{17} \mathrm{H}_{19}$ & 110.477 & & 110.478 & \\
\hline $\mathrm{C}_{14} \mathrm{C}_{17} \mathrm{H}_{20}$ & 112.028 & & 112.001 & \\
\hline $\mathrm{C}_{9} \mathrm{C}_{12} \mathrm{C}_{21}$ & 111.382 & & 111.384 & \\
\hline $\mathrm{C}_{12} \mathrm{C}_{21} \mathrm{H}_{22}$ & 109.228 & & 109.231 & \\
\hline $\mathrm{C}_{12} \mathrm{C}_{21} \mathrm{H}_{23}$ & 109.005 & & 109.019 & \\
\hline $\mathrm{C}_{12} \mathrm{C}_{21} \mathrm{C}_{24}$ & 114.775 & & 114.824 & \\
\hline $\mathrm{C}_{21} \mathrm{C}_{24} \mathrm{H}_{25}$ & 108.695 & & 108.731 & \\
\hline $\mathrm{C}_{21} \mathrm{C}_{24} \mathrm{H}_{26}$ & 110.286 & & 110.291 & \\
\hline $\mathrm{C}_{21} \mathrm{C}_{24} \mathrm{C}_{27}$ & 113.287 & & 113.308 & \\
\hline $\mathrm{C}_{24} \mathrm{C}_{27} \mathrm{H}_{28}$ & 109.207 & & 109.229 & \\
\hline $\mathrm{C}_{24} \mathrm{C}_{27} \mathrm{H}_{29}$ & 109.217 & & 109.208 & \\
\hline $\mathrm{C}_{24} \mathrm{C}_{27} \mathrm{C}_{30}$ & 113.280 & & 109.208 & \\
\hline $\mathrm{C}_{27} \mathrm{C}_{30} \mathrm{H}_{31}$ & 111.428 & & 111.428 & \\
\hline $\mathrm{C}_{27} \mathrm{C}_{30} \mathrm{H}_{32}$ & 111.218 & & 111.209 & \\
\hline $\mathrm{C}_{27} \mathrm{C}_{30} \mathrm{H}_{33}$ & 111.189 & & 111.202 & \\
\hline \multicolumn{5}{|l|}{ Dihedral angles $\left({ }^{\circ}\right)$} \\
\hline $\mathrm{H}_{3} \mathrm{H}_{2} \mathrm{C}_{1} \mathrm{C}_{4}$ & 180 & & -179.908 & \\
\hline $\mathrm{H}_{2} \mathrm{C}_{1} \mathrm{C}_{4} \mathrm{H}_{5}$ & -179.977 & & 179.808 & \\
\hline $\mathrm{H}_{2} \mathrm{C}_{1} \mathrm{C}_{4} \mathrm{C}_{6}$ & 0.037 & & 0.106 & \\
\hline $\mathrm{C}_{1} \mathrm{C}_{4} \mathrm{C}_{6} \mathrm{O}_{7}$ & -0.166 & & 178.652 & \\
\hline $\mathrm{C}_{1} \mathrm{C}_{4} \mathrm{C}_{6} \mathrm{O}_{8}$ & 179.844 & & -1.298 & \\
\hline $\mathrm{C}_{4} \mathrm{C}_{6} \mathrm{O}_{8} \mathrm{C}_{9}$ & 179.896 & & 179.515 & \\
\hline $\mathrm{C}_{6} \mathrm{O}_{8} \mathrm{C}_{9} \mathrm{H}_{10}$ & 55.606 & & 55.506 & \\
\hline $\mathrm{C}_{6} \mathrm{O}_{8} \mathrm{C}_{9} \mathrm{H}_{11}$ & -61.282 & & -61.454 & \\
\hline $\mathrm{C}_{6} \mathrm{O}_{8} \mathrm{C}_{9} \mathrm{C}_{12}$ & 177.303 & & 177.169 & \\
\hline $\mathrm{O}_{8} \mathrm{C}_{9} \mathrm{C}_{12} \mathrm{H}_{13}$ & 55.024 & & 54.463 & \\
\hline $\mathrm{O}_{8} \mathrm{C}_{9} \mathrm{C}_{12} \mathrm{C}_{14}$ & 171.114 & & 170.547 & \\
\hline $\mathrm{C}_{9} \mathrm{C}_{12} \mathrm{C}_{14} \mathrm{H}_{15}$ & 61.844 & & 61.568 & \\
\hline $\mathrm{C}_{9} \mathrm{C}_{12} \mathrm{C}_{14} \mathrm{H}_{16}$ & -53.102 & & -53.378 & \\
\hline $\mathrm{C}_{9} \mathrm{C}_{12} \mathrm{C}_{14} \mathrm{C}_{17}$ & -175.215 & & -175.516 & \\
\hline $\mathrm{C}_{12} \mathrm{C}_{14} \mathrm{C}_{17} \mathrm{H}_{18}$ & 56.957 & & 57.249 & \\
\hline $\mathrm{C}_{12} \mathrm{C}_{14} \mathrm{C}_{17} \mathrm{H}_{19}$ & 176.491 & & 176.782 & \\
\hline $\mathrm{C}_{12} \mathrm{C}_{14} \mathrm{C}_{17} \mathrm{H}_{20}$ & -63.865 & & -63.596 & \\
\hline $\mathrm{O}_{8} \mathrm{C}_{9} \mathrm{C}_{12} \mathrm{C}_{21}$ & -63.322 & & -63.915 & \\
\hline $\mathrm{C}_{9} \mathrm{C}_{12} \mathrm{C}_{21} \mathrm{H}_{22}$ & -96.883 & & -97.147 & \\
\hline $\mathrm{C}_{9} \mathrm{C}_{12} \mathrm{C}_{21} \mathrm{H}_{23}$ & 18.587 & & 18.279 & \\
\hline $\mathrm{C}_{9} \mathrm{C}_{12} \mathrm{C}_{21} \mathrm{C}_{24}$ & 139.536 & & 139.298 & \\
\hline $\mathrm{C}_{12} \mathrm{C}_{21} \mathrm{C}_{24} \mathrm{H}_{25}$ & -59.615 & & -59.061 & \\
\hline $\mathrm{C}_{12} \mathrm{C}_{21} \mathrm{C}_{24} \mathrm{H}_{26}$ & 56.214 & & 56.717 & \\
\hline
\end{tabular}


TABle 1: Continued.

\begin{tabular}{|c|c|c|c|c|}
\hline Internal coordinates & $\begin{array}{c}\text { Level of theory } \\
\text { DFT/6-311+G** } \\
\text { s-cis EHA }\end{array}$ & $\begin{array}{l}\text { s-cis methyl } \\
\text { acrylate }^{\mathrm{a}}\end{array}$ & $\begin{array}{c}\mathrm{DFT} / 6-311+\mathrm{G}^{* *} \\
\text { s-trans EHA }\end{array}$ & $\begin{array}{l}\text { s-trans methyl } \\
\text { acrylate }^{\mathrm{b}}\end{array}$ \\
\hline $\mathrm{C}_{12} \mathrm{C}_{21} \mathrm{C}_{24} \mathrm{C}_{27}$ & 178.823 & & 179.360 & \\
\hline $\mathrm{C}_{21} \mathrm{C}_{24} \mathrm{C}_{27} \mathrm{H}_{28}$ & -58.771 & & -58.768 & \\
\hline $\mathrm{C}_{21} \mathrm{C}_{24} \mathrm{C}_{27} \mathrm{H}_{29}$ & 56.758 & & 56.753 & \\
\hline $\mathrm{C}_{21} \mathrm{C}_{24} \mathrm{C}_{27} \mathrm{C}_{30}$ & 179.033 & & 179.029 & \\
\hline $\mathrm{C}_{24} \mathrm{C}_{27} \mathrm{C}_{30} \mathrm{H}_{31}$ & 179.693 & & -179.983 & \\
\hline $\mathrm{C}_{24} \mathrm{C}_{27} \mathrm{C}_{30} \mathrm{H}_{32}$ & -60.205 & & -59.888 & \\
\hline $\mathrm{C}_{24} \mathrm{C}_{27} \mathrm{C}_{30} \mathrm{H}_{33}$ & 59.599 & & 59.917 & \\
\hline
\end{tabular}

${ }^{a}$ Determined by a joint analysis of electron diffraction data and rotational constants [21].

${ }^{\mathrm{b}}$ Determined by ab initio calculation at $\mathrm{HF} / 6-31 \mathrm{G}^{* *}[20]$.

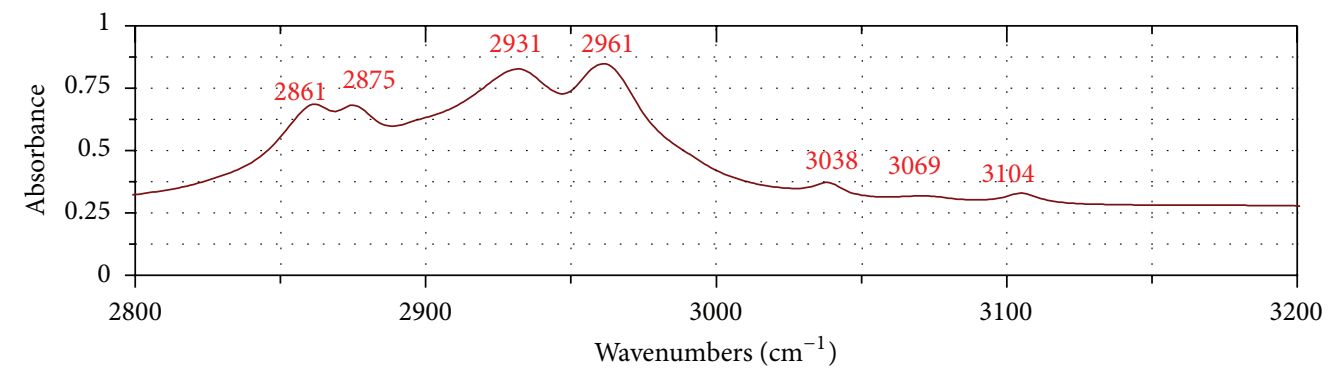

(a)

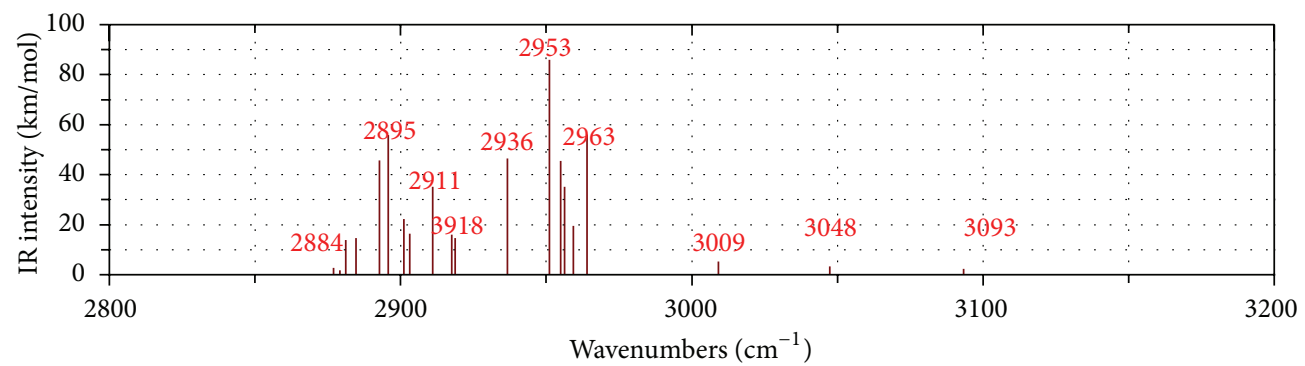

(b)

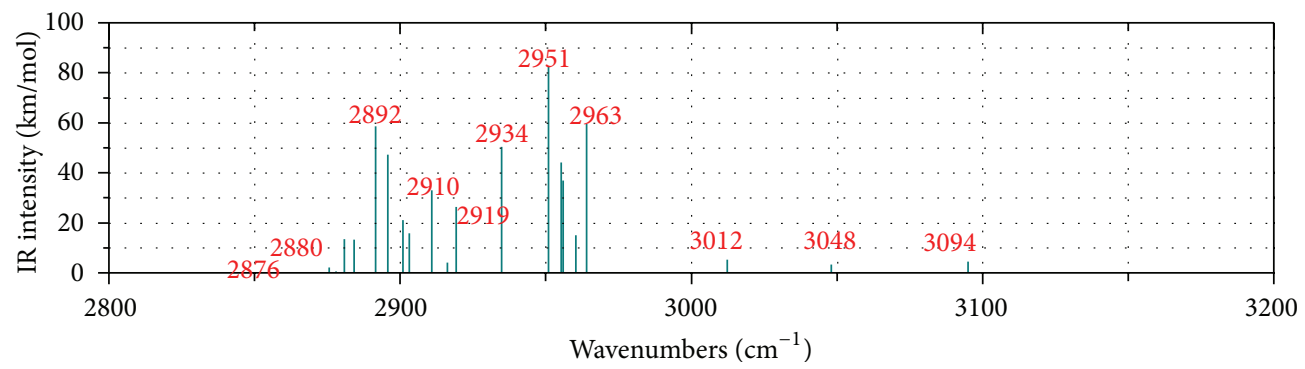

(c)

FIGURE 2: Comparison of the experimental IR (a) CH stretching region (3200-2800 $\mathrm{cm}^{-1}$ ) with the appropriate theoretical DFT//B3LYP/6$311+\mathrm{G}^{* *}$ spectra ((b) and (c)) for s-cis and s-trans 2-ethylhexyl acrylate, respectively.

the butyl and the ethyl sides of the 2-ethylhexyl acrylate molecule.

\subsection{The $C=O$ and $C=C$ Stretching and $C H$ Bending Vibrations} (1800-1200 $\mathrm{cm}^{-1}$, Figure 3). In the IR spectrum (Figure 3(a)), a very strong peak at $1727 \mathrm{~cm}^{-1}$ represents the $\mathrm{C}=\mathrm{O}$ stretching band. The simulated peaks are at 1735 and $1731 \mathrm{~cm}^{-1}$ for the s-cis and s-trans conformations; these two frequencies contribute to the experimental $\mathrm{C}=\mathrm{O}$ stretching band.

There are two $\mathrm{C}=\mathrm{C}$ stretching bands in the experimental spectra located at 1637 and $1619 \mathrm{~cm}^{-1}$, of medium intensity. These bands are well reproduced by the theoretical bands at 
TABLE 2: Frequencies and PEDs of s-cis 2-ethylhexyl acrylate.

\begin{tabular}{|c|c|c|c|c|c|}
\hline Number ${ }^{\mathrm{b}}$ & $\begin{array}{l}\text { Observed } \\
\left(\mathrm{cm}^{-1}\right)\end{array}$ & $\begin{array}{l}\text { DFT/6-311+G }{ }^{* *} \\
\text { Unscaled }\left(\mathrm{cm}^{-1}\right)\end{array}$ & Scaled $\left(\mathrm{cm}^{-1}\right)$ & $\begin{array}{c}\text { Intensity } \\
\left(\mathrm{km} \cdot \mathrm{mol}^{-1}\right)\end{array}$ & Assignment $(\% \mathrm{PED})^{\mathrm{a}}$ \\
\hline 1 & - & 30.5017 & 30,7519 & 0.1860 & $\tau\left(\mathrm{O}_{8} \mathrm{C}_{9}\right)(63) ; \tau\left(\mathrm{C}_{9} \mathrm{C}_{12}\right)(35)$ \\
\hline 2 & - & 36.7273 & 37,02484 & 1.9384 & $\tau\left(\mathrm{C}_{9} \mathrm{C}_{12}\right)(83) ; \tau\left(\mathrm{O}_{8} \mathrm{C}_{9}\right)(17)$ \\
\hline 3 & - & 48.2766 & 48,65862 & 1.2435 & $\tau\left(\mathrm{C}_{9} \mathrm{C}_{12}\right)(98)$ \\
\hline 4 & - & 74.7391 & 75,29828 & 0.0096 & $\begin{array}{l}\rho\left(\mathrm{C}_{30} \mathrm{H}_{3}\right)(62) ; \tau\left(\mathrm{C}_{9} \mathrm{C}_{12}\right)(17) ; \tau\left(\mathrm{O}_{8} \mathrm{C}_{9}\right)(7) ; \rho\left(\mathrm{C}_{14} \mathrm{H}_{2}\right) \\
(7)\end{array}$ \\
\hline 5 & - & 93.1493 & 93,81827 & 0.7602 & $\tau\left(\mathrm{C}_{9} \mathrm{C}_{12}\right)(84) ; \rho\left(\mathrm{C}_{14} \mathrm{H}_{2}\right)(15)$ \\
\hline 6 & - & 99.6177 & 100,32262 & 0.6102 & $\tau\left(\mathrm{C}_{9} \mathrm{C}_{12}\right)(76) ; \tau\left(\mathrm{C}_{9} \mathrm{C}_{12}\right)(21)$ \\
\hline 7 & - & 115.9962 & 116,78605 & 0.2949 & $\tau\left(\mathrm{C}_{9} \mathrm{C}_{12}\right)(94)$ \\
\hline 8 & - & 133.8201 & 134,69244 & 0.7707 & $\tau\left(\mathrm{C}_{9} \mathrm{C}_{12}\right)(43) ; \rho\left(\mathrm{C}_{27} \mathrm{H}_{3}\right)(31) ; \rho\left(\mathrm{C}_{9} \mathrm{H}_{2}\right)(15)$ \\
\hline 9 & - & 146.1924 & 147,11591 & 0.0469 & $\tau\left(\mathrm{C}_{9} \mathrm{C}_{12}\right)(58) ; \rho\left(\mathrm{C}_{9} \mathrm{H}_{2}\right)(17) ; \rho\left(\mathrm{C}_{14} \mathrm{H}_{2}\right)(16)$ \\
\hline 10 & - & 201.0708 & 202,16112 & 1.8808 & $\tau\left(\mathrm{C}_{9} \mathrm{C}_{12}\right)(89) ; \delta\left(\mathrm{C}_{6} \mathrm{O}_{8} \mathrm{C}_{9}\right)(5)$ \\
\hline 11 & - & 213.9423 & 215,05753 & 2.7336 & $\tau\left(\mathrm{C}_{9} \mathrm{C}_{12}\right)(98)$ \\
\hline 12 & - & 234.1345 & 235,27792 & 0.6986 & $\rho\left(\mathrm{C}_{30} \mathrm{H}_{3}\right)(94)$ \\
\hline 13 & - & 253.7618 & 254,91989 & 1.2866 & $\rho\left(\mathrm{C}_{30} \mathrm{H}_{3}\right)(99)$ \\
\hline 14 & - & 265.4468 & 266,60766 & 0.7334 & $\tau\left(\mathrm{C}_{9} \mathrm{C}_{12}\right)(83) ; \rho\left(\mathrm{C}_{30} \mathrm{H}_{3}\right)(14)$ \\
\hline 15 & - & 285.1163 & 286,27176 & 3.5548 & $\rho\left(\mathrm{C}_{30} \mathrm{H}_{3}\right)(78) ; \tau\left(\mathrm{C}_{9} \mathrm{C}_{12}\right)(18)$ \\
\hline 16 & - & 315.7568 & 316,87874 & 0.5814 & $\rho\left(\mathrm{C}_{9} \mathrm{H}_{2}\right)(89) ; \rho\left(\mathrm{C}_{17} \mathrm{H}_{3}\right)(6)$ \\
\hline 17 & - & 355.1126 & 356,14657 & 0.2263 & $\rho\left(\mathrm{C}_{17} \mathrm{H}_{3}\right)(36) ; \rho\left(\mathrm{C}_{9} \mathrm{C}_{12} \mathrm{C}_{14}\right)(31) ; \rho\left(\mathrm{C}_{9} \mathrm{H}_{2}\right)(19)$ \\
\hline 18 & - & 375.9666 & 376,93349 & 4.3994 & $\begin{array}{l}\rho\left(\mathrm{C}_{24} \mathrm{C}_{27} \mathrm{C}_{30}\right)(27) ; \rho\left(\mathrm{C}_{9} \mathrm{C}_{12} \mathrm{C}_{14}\right)(23) ; \rho\left(\mathrm{C}_{17} \mathrm{H}_{3}\right)(17) ; \delta \\
\left(\mathrm{C}_{21} \mathrm{C}_{24} \mathrm{C}_{27}\right)(10) ; \rho\left(\mathrm{C}_{9} \mathrm{C}_{12} \mathrm{H}_{13}\right)(10) ; \delta\left(\mathrm{C}_{21} \mathrm{H}_{2}\right)(8)\end{array}$ \\
\hline 19 & - & 470.318 & 470,80422 & 0.6863 & $\rho\left(\mathrm{C}_{9} \mathrm{C}_{12} \mathrm{C}_{21}\right)(72) ; \rho\left(\mathrm{C}_{17} \mathrm{H}_{3}\right)(11) ; \rho\left(\mathrm{C}_{9} \mathrm{H}_{2}\right)(5)$ \\
\hline 20 & - & 512.0856 & 512,26637 & 6.2054 & $\rho\left(\mathrm{C}_{9} \mathrm{C}_{12} \mathrm{C}_{14}\right)(80) ; \rho\left(\mathrm{C}_{9} \mathrm{H}_{2}\right)(7) ; \delta\left(\mathrm{C}_{21} \mathrm{C}_{12} \mathrm{C}_{14}\right)(6)$ \\
\hline 21 & - & 527.3488 & 527,40376 & 0.1504 & $\rho\left(\mathrm{C}_{9} \mathrm{H}_{2}\right)(87) ; \mathrm{O}\left(\mathrm{C}_{6} \mathrm{O}_{7}\right)(9)$ \\
\hline 22 & - & 533.9978 & 533,99558 & 1.1100 & $\rho\left(\mathrm{C}_{9} \mathrm{C}_{12} \mathrm{C}_{14}\right)(77) ; \rho\left(\mathrm{C}_{6} \mathrm{O}_{8} \mathrm{C}_{9}\right)(8)$ \\
\hline 23 & - & 681.189 & 679,55184 & 3.1928 & $\rho\left(\mathrm{C}_{9} \mathrm{C}_{12} \mathrm{C}_{14}\right)(42) ; \delta\left(\mathrm{O}_{7} \mathrm{C}_{6} \mathrm{C}_{4}\right)(42) ; \rho\left(\mathrm{C}_{6} \mathrm{O}_{8} \mathrm{C}_{9}\right)(5)$ \\
\hline 24 & $731(\mathrm{~m})$ & 731.2122 & 728,8586 & 4.5137 & $\rho\left(\mathrm{C}_{21} \mathrm{C}_{24} \mathrm{C}_{27}\right)(99)$ \\
\hline 25 & $748(\mathrm{sh})$ & 779.5982 & 776,474 & 1.0039 & $\rho\left(\mathrm{C}_{27} \mathrm{H}_{2}\right)(65) ; \rho\left(\mathrm{C}_{9} \mathrm{C}_{12} \mathrm{C}_{14}\right)(18) ; \rho\left(\mathrm{C}_{14} \mathrm{H}_{2}\right)(12)$ \\
\hline 26 & $770(\mathrm{~m})$ & 786.3101 & 783,07298 & 1.6507 & $\begin{array}{l}\rho\left(\mathrm{C}_{27} \mathrm{H}_{2}\right)(36) ; \rho\left(\mathrm{C}_{21} \mathrm{H}_{2}\right)(32) ; \rho\left(\mathrm{C}_{14} \mathrm{H}_{2}\right)(23) ; \rho \\
\left(\mathrm{C}_{17} \mathrm{H}_{3}\right)(8)\end{array}$ \\
\hline 27 & $811(\mathrm{~m})$ & 829.5925 & 825,59191 & 24.9487 & $\rho\left(\mathrm{C}_{9} \mathrm{H}_{2}\right)(73) ; \mathrm{O}\left(\mathrm{C}_{6} \mathrm{O}_{7}\right)(21) ; \mathrm{y}\left(\mathrm{C}_{1} \mathrm{H}_{2}\right)(8)$ \\
\hline 28 & $852(w)$ & 852.3514 & 847,92486 & 3.0736 & $\rho\left(\mathrm{C}_{9} \mathrm{C}_{12} \mathrm{C}_{21}\right)(82) ; \rho\left(\mathrm{C}_{9} \mathrm{H}_{2}\right)(16)$ \\
\hline 29 & $896(w)$ & 901.6472 & 896,24016 & 1.0870 & $\begin{array}{l}\rho\left(\mathrm{C}_{9} \mathrm{C}_{12} \mathrm{H}_{21}\right)(62) ; \omega\left(\mathrm{C}_{24} \mathrm{H}_{2}\right)(16) ; \mathrm{y}\left(\mathrm{C}_{21} \mathrm{H}_{2}\right)(9) ; \delta \\
\left(\mathrm{C}_{30} \mathrm{H}_{3}\right)(8)\end{array}$ \\
\hline 30 & - & 906.8585 & 901,34317 & 1.0553 & $\rho\left(\mathrm{C}_{9} \mathrm{C}_{12} \mathrm{H}_{21}\right)(86) ; \rho\left(\mathrm{C}_{9} \mathrm{H}_{2}\right)(10)$ \\
\hline 31 & $909(\mathrm{w})$ & 917.2167 & 911,48352 & 7.2928 & $\rho\left(\mathrm{C}_{9} \mathrm{H}_{2}\right)(58) ; \omega\left(\mathrm{C}_{27} \mathrm{H}_{2}\right)(27) ; \rho\left(\mathrm{O}_{7} \mathrm{C}_{6} \mathrm{O}_{8}\right)(12)$ \\
\hline 32 & 933 (sh) & 947.8927 & 941,49381 & 5.9570 & $\delta\left(\mathrm{C}_{14} \mathrm{C}_{12} \mathrm{H}_{3}\right)(50) ; \omega\left(\mathrm{C}_{27} \mathrm{H}_{2}\right)$ \\
\hline 33 & $962(\mathrm{~m})$ & 983.6705 & 976,45643 & 25.1442 & $\begin{array}{l}\rho\left(\mathrm{C}_{9} \mathrm{C}_{12} \mathrm{H}_{13}\right)(83) ; \delta\left(\mathrm{C}_{6} \mathrm{O}_{8} \mathrm{C}_{9}\right)(21) ; \mathrm{y}\left(\mathrm{C}_{21} \mathrm{H}_{2}\right)(14) ; \mathrm{y} \\
\left(\mathrm{C}_{27} \mathrm{H}_{2}\right)(11) ; \mathrm{y}\left(\mathrm{C}_{14} \mathrm{H}_{2}\right)(11)\end{array}$ \\
\hline 34 & $984(\mathrm{~m})$ & 1006.9955 & 999,22751 & 25.5334 & $\omega\left(\mathrm{C}_{1} \mathrm{H}_{2}\right)(84) ; \mathrm{y}\left(\mathrm{C}_{14} \mathrm{H}_{2}\right)(10)$ \\
\hline 35 & - & 1016.9241 & 1008,91495 & 5.1103 & $\begin{array}{l}\rho\left(\mathrm{C}_{14} \mathrm{H}_{2}\right)(41) ; \rho\left(\mathrm{C}_{9} \mathrm{C}_{12} \mathrm{C}_{21}\right)(24) ; \delta\left(\mathrm{C}_{12} \mathrm{C}_{21} \mathrm{C}_{24}\right)(23) ; \rho \\
\left(\mathrm{C}_{17} \mathrm{H}_{3}\right)(7)\end{array}$ \\
\hline 36 & $1015(\mathrm{sh})$ & 1023.2765 & 1015,11136 & 26.0306 & o $\left(\mathrm{C}_{4} \mathrm{H}_{5}\right)(99)$ \\
\hline 37 & - & 1025.8451 & 1017,61651 & 1.9504 & $\delta\left(\mathrm{C}_{9} \mathrm{C}_{12} \mathrm{C}_{14}\right)(98) ; \nu\left(\mathrm{C}_{9} \mathrm{O}_{8}\right)(5)$ \\
\hline 38 & $1025(\mathrm{sh})$ & 1045.9956 & 1037,26182 & 27.8636 & $\mathrm{y}\left(\mathrm{C}_{24} \mathrm{H}_{2}\right)(42) ; \nu\left(\mathrm{C}_{9} \mathrm{C}_{12}\right)(42) ; \nu\left(\mathrm{O}_{8} \mathrm{C}_{9}\right)(6)$ \\
\hline 39 & - & 1062.1068 & 1052,95957 & 0.4109 & $\rho\left(\mathrm{C}_{14} \mathrm{C}_{12} \mathrm{H}_{13}\right)(83) ; \nu\left(\mathrm{C}_{27} \mathrm{C}_{30}\right)(12)$ \\
\hline 40 & - & 1065.9864 & 1056,73835 & 0.4863 & $v\left(\mathrm{C}_{14} \mathrm{C}_{17}\right)(83) ; \nu\left(\mathrm{C}_{24} \mathrm{C}_{27}\right)(11)$ \\
\hline 41 & - & 1083.5238 & 1073,81387 & 1.6531 & $\omega\left(\mathrm{C}_{9} \mathrm{H}_{2}\right)(83) ; \omega\left(\mathrm{C}_{21} \mathrm{H}_{2}\right)(15)$ \\
\hline 42 & $1057(\mathrm{~m})$ & 1089.4787 & 1079,60965 & 2.2238 & $\rho\left(\mathrm{C}_{9} \mathrm{H}_{2}\right)(84) ; \omega\left(\mathrm{C}_{27} \mathrm{H}_{2}\right)(14)$ \\
\hline
\end{tabular}


TABLE 2: Continued.

\begin{tabular}{|c|c|c|c|c|c|}
\hline Number ${ }^{b}$ & $\begin{array}{c}\text { Observed } \\
\left(\mathrm{cm}^{-1}\right)\end{array}$ & $\begin{array}{l}\text { DFT/6-311+G } \mathrm{G}^{* *} \\
\text { Unscaled }\left(\mathrm{cm}^{-1}\right)\end{array}$ & Scaled $\left(\mathrm{cm}^{-1}\right)$ & $\begin{array}{c}\text { Intensity } \\
\left(\mathrm{km} \cdot \mathrm{mol}^{-1}\right)\end{array}$ & Assignment $(\% \mathrm{PED})^{\mathrm{a}}$ \\
\hline 43 & - & 1150.5508 & 1138,98319 & 1.9252 & $\rho\left(\mathrm{C}_{9} \mathrm{C}_{12} \mathrm{H}_{13}\right)(96)$ \\
\hline 44 & $1116(w)$ & 1168.5589 & 1156,46723 & 11.8316 & $\begin{array}{l}\delta\left(\mathrm{C}_{12} \mathrm{C}_{21} \mathrm{C}_{24}\right)(67) ; \rho\left(\mathrm{C}_{14} \mathrm{C}_{12} \mathrm{H}_{13}\right)(9) ; \rho\left(\mathrm{O}_{8} \mathrm{C}_{9} \mathrm{C}_{12}\right)(7) ; \\
\delta\left(\mathrm{C}_{14} \mathrm{C}_{12} \mathrm{C}_{21}\right)(67)\end{array}$ \\
\hline 45 & $1060(\mathrm{sh})$ & 1185.6606 & 1173,06145 & 0.0857 & $\delta\left(\mathrm{C}_{9} \mathrm{C}_{12} \mathrm{C}_{14}\right)(63) ; \rho\left(\mathrm{C}_{30} \mathrm{H}_{3}\right)(26) ; \delta\left(\mathrm{C}_{12} \mathrm{C}_{14} \mathrm{C}_{17}\right)(6)$ \\
\hline 46 & 1191 & 1205.1044 & 1191,9167 & 626.1232 & $\rho\left(\mathrm{C}_{6} \mathrm{C}_{4} \mathrm{H}_{5}\right)(87)$ \\
\hline 47 & - & 1243.7853 & 1229,3901 & 0.3621 & $\mathrm{y}\left(\mathrm{C}_{21} \mathrm{H}_{2}\right)(94) ; \mathrm{y}\left(\mathrm{C}_{27} \mathrm{H}_{2}\right)(5)$ \\
\hline 48 & - & 1258.6429 & 1243,77093 & 1.0505 & $\rho\left(\mathrm{C}_{9} \mathrm{H}_{2}\right)(76) ; \omega\left(\mathrm{C}_{21} \mathrm{H}_{2}\right)(23)$ \\
\hline 49 & - & 1281.7118 & 1266,0853 & 0.7178 & $\mathrm{y}\left(\mathrm{C}_{14} \mathrm{H}_{2}\right)(55) ; \omega\left(\mathrm{C}_{21} \mathrm{H}_{2}\right)(44)$ \\
\hline 50 & - & 1300.3301 & 1284,08198 & 1.3337 & $\omega\left(\mathrm{C}_{21} \mathrm{H}_{2}\right)(93)$ \\
\hline 51 & - & 1317.9028 & 1301,05761 & 11.1581 & $\begin{array}{l}\omega\left(\mathrm{C}_{24} \mathrm{H}_{2}\right)(24) ; \rho\left(\mathrm{C}_{9} \mathrm{C}_{12} \mathrm{H}_{13}\right)(17) ; \mathrm{y}\left(\mathrm{C}_{14} \mathrm{H}_{2}\right)(13) ; \omega \\
\left(\mathrm{C}_{27} \mathrm{H}_{2}\right)(7) ; \rho\left(\mathrm{C}_{6} \mathrm{O}_{8} \mathrm{C}_{9}\right)(5)\end{array}$ \\
\hline 52 & 1272 & 1324.5992 & 1307,52384 & 68.3227 & $\begin{array}{l}\mathrm{y}\left(\mathrm{C}_{27} \mathrm{H}_{2}\right)(37) ; \rho\left(\mathrm{C}_{9} \mathrm{C}_{12} \mathrm{H}_{13}\right)(29) ; \mathrm{y}\left(\mathrm{C}_{21} \mathrm{H}_{2}\right)(22) ; \mathrm{y} \\
\left(\mathrm{C}_{14} \mathrm{H}_{2}\right)(6)\end{array}$ \\
\hline 53 & 1295 & 1328.2386 & 1311,03753 & 6.0034 & $\mathrm{y}\left(\mathrm{C}_{27} \mathrm{H}_{2}\right)(93)$ \\
\hline 54 & - & 1338.1682 & 1320,62195 & 0.5711 & $\mathrm{y}\left(\mathrm{C}_{24} \mathrm{H}_{2}\right)(70) ; \mathrm{y}\left(\mathrm{C}_{27} \mathrm{H}_{2}\right)(28)$ \\
\hline 55 & 1340 & 1361.0156 & 1342,66291 & 1.3124 & $\omega\left(\mathrm{C}_{21} \mathrm{H}_{2}\right)(78) ; \omega\left(\mathrm{C}_{9} \mathrm{H}_{2}\right)(21)$ \\
\hline 56 & - & 1381.2224 & 1362,1423 & 0.4732 & $\rho\left(\mathrm{C}_{9} \mathrm{C}_{12} \mathrm{C}_{21}\right)(78) ; \omega\left(\mathrm{C}_{21} \mathrm{H}_{2}\right)(21)$ \\
\hline 57 & 1357 & 1394.2309 & 1374,67547 & 3.3349 & $\omega\left(\mathrm{C}_{9} \mathrm{H}_{2}\right)(95)$ \\
\hline 58 & - & 1404.5719 & 1384,63467 & 4.5032 & $\omega\left(\mathrm{C}_{24} \mathrm{H}_{2}\right)(95) ; \omega\left(\mathrm{C}_{21} \mathrm{H}_{2}\right)(5)$ \\
\hline 59 & 1381 & 1410.9338 & 1390,75996 & 7.3272 & $\omega\left(\mathrm{C}_{21} \mathrm{H}_{2}\right)(97)$ \\
\hline 60 & - & 1414.1585 & 1393,86422 & 2.3703 & $\omega\left(\mathrm{C}_{21} \mathrm{H}_{2}\right)(99)$ \\
\hline 61 & - & 1418.0649 & 1397,62426 & 0.4645 & $\omega\left(\mathrm{C}_{21} \mathrm{H}_{2}\right)(93) ; \omega\left(\mathrm{C}_{14} \mathrm{H}_{2}\right)(6)$ \\
\hline 62 & 1408 & 1437.761 & 1416,57487 & 83.1633 & $\omega\left(\mathrm{C}_{9} \mathrm{H}_{2}\right)(49) ; \omega\left(\mathrm{C}_{21} \mathrm{H}_{2}\right)(26) ; \delta\left(\mathrm{CH}_{2}\right)(20)$ \\
\hline 63 & - & 1486.1635 & 1463,09161 & 1.0310 & $\begin{array}{l}\delta\left(\mathrm{C}_{21} \mathrm{H}_{2}\right)(40) ; \delta\left(\mathrm{C}_{24} \mathrm{H}_{2}\right)(20) ; \delta\left(\mathrm{C}_{12} \mathrm{C}_{21} \mathrm{C}_{24}\right)(12) ; \delta \\
\left(\mathrm{C}_{14} \mathrm{H}_{2}\right)(7)\end{array}$ \\
\hline 64 & - & 1488.3957 & 1465,235 & 1.0310 & $\begin{array}{l}\delta\left(\mathrm{C}_{27} \mathrm{H}_{2}\right)(29) ; \delta\left(\mathrm{C}_{21} \mathrm{H}_{2}\right)(28) ; \delta\left(\mathrm{C}_{24} \mathrm{H}_{2}\right)(18) ; \delta \\
\left(\mathrm{C}_{12} \mathrm{C}_{21} \mathrm{C}_{24}\right)(14) ; \delta\left(\mathrm{C}_{14} \mathrm{H}_{2}\right)(10) ; \delta\left(\mathrm{C}_{27} \mathrm{H}_{2}\right)(10)\end{array}$ \\
\hline 65 & - & 1492.293 & 1468,97685 & 1.3617 & $\delta\left(\mathrm{C}_{21} \mathrm{H}_{2}\right)(67) ; \delta\left(\mathrm{C}_{27} \mathrm{H}_{2}\right)(11) ; \delta\left(\mathrm{C}_{14} \mathrm{H}_{2}\right)(7)$ \\
\hline 66 & - & 1499.9113 & 1476,28987 & 8.3925 & $\delta\left(\mathrm{C}_{30} \mathrm{H}_{3}\right)(88) ; \delta\left(\mathrm{C}_{27} \mathrm{H}_{2}\right)(10)$ \\
\hline 67 & - & 1501.7672 & 1478,07111 & 4.3738 & $\begin{array}{l}\delta\left(\mathrm{C}_{21} \mathrm{H}_{2}\right)(29) ; \delta\left(\mathrm{C}_{30} \mathrm{H}_{3}\right)(28) ; \delta\left(\mathrm{C}_{24} \mathrm{H}_{2}\right)(15) ; \delta \\
\left(\mathrm{C}_{21} \mathrm{C}_{12} \mathrm{H}_{13}\right)(11) ; \delta\left(\mathrm{C}_{14} \mathrm{H}_{2}\right)(6)\end{array}$ \\
\hline 68 & - & 1503.707 & 1479,93276 & 3.5078 & $\delta\left(\mathrm{C}_{27} \mathrm{H}_{2}\right)(31) ; \delta\left(\mathrm{C}_{21} \mathrm{H}_{2}\right)(29) ; \delta\left(\mathrm{C}_{9} \mathrm{H}_{2}\right)(18)$ \\
\hline 69 & 1464 & 1509.3473 & 1485,34511 & 9.9123 & $\begin{array}{l}\delta\left(\mathrm{C}_{17} \mathrm{H}_{3}\right)(27) ; \delta\left(\mathrm{C}_{27} \mathrm{H}_{2}\right)(26) ; \delta\left(\mathrm{C}_{21} \mathrm{H}_{2}\right)(10) ; \delta \\
\left(\mathrm{C}_{12} \mathrm{C}_{21} \mathrm{C}_{24}\right)(8) ; \delta\left(\mathrm{C}_{24} \mathrm{H}_{2}\right)(7) ; \delta\left(\mathrm{C}_{12} \mathrm{C}_{14} \mathrm{C}_{21}\right)(6)\end{array}$ \\
\hline 70 & - & 1510.6836 & 1486,62726 & 10.8575 & $\delta\left(\mathrm{C}_{21} \mathrm{H}_{2}\right)(83) ; \delta\left(\mathrm{C}_{27} \mathrm{H}_{2}\right)(10)$ \\
\hline 71 & - & 1515.4657 & 1491,21508 & 6.0184 & $\begin{array}{l}\delta\left(\mathrm{C}_{24} \mathrm{H}_{2}\right)(42) ; \delta\left(\mathrm{C}_{21} \mathrm{C}_{24} \mathrm{C}_{27}\right)(25) ; \delta\left(\mathrm{C}_{27} \mathrm{H}_{2}\right)(13) ; \delta \\
\left(\mathrm{C}_{21} \mathrm{H}_{2}\right)(10)\end{array}$ \\
\hline 72 & 1637 & 1687.5503 & 1655,81242 & 26.9151 & $\nu\left(\mathrm{C}_{1} \mathrm{C}_{4}\right)(87) ; \delta\left(\mathrm{C}_{1} \mathrm{H}_{2}\right)(7)$ \\
\hline 73 & 1727 & 1770.955 & 1735,24092 & 243.4946 & $\begin{array}{l}\nu\left(\mathrm{C}_{6} \mathrm{O}_{7}\right)(80) ; \delta\left(\mathrm{O}_{8} \mathrm{C}_{9} \mathrm{H}_{12}\right)(7) ; \delta\left(\mathrm{C}_{21} \mathrm{H}_{2}\right)(5) ; \nu\left(\mathrm{C}_{4} \mathrm{C}_{6}\right) \\
(5)\end{array}$ \\
\hline 74 & - & 2997.3914 & 2877,02371 & 2.7860 & $v\left(\mathrm{C}_{21} \mathrm{H}_{22}\right)(45) ; v\left(\mathrm{C}_{14} \mathrm{H}_{2}\right)(33) ; v\left(\mathrm{C}_{12} \mathrm{H}_{13}\right)(14)$ \\
\hline 75 & - & 2999.8142 & 2879,23075 & 1.7988 & $v\left(\mathrm{C}_{21} \mathrm{H}_{2}\right)(76) ; v\left(\mathrm{C}_{24} \mathrm{H}_{2}\right)(14)$ \\
\hline 76 & - & 3002.0159 & 2881,23622 & 13.8469 & $\begin{array}{l}v\left(\mathrm{C}_{27} \mathrm{H}_{2}\right)(33) ; v\left(\mathrm{C}_{24} \mathrm{H}_{2}\right)(32) ; v\left(\mathrm{C}_{30} \mathrm{H}_{31}\right)(22) ; v \\
\left(\mathrm{C}_{21} \mathrm{H}_{2}\right)(6)\end{array}$ \\
\hline 77 & - & 3005.8916 & 2884,76609 & 14.6381 & $v\left(\mathrm{C}_{21} \mathrm{H}_{2}\right)(74) ; v\left(\mathrm{C}_{14} \mathrm{H}_{2}\right)(14) ; v\left(\mathrm{C}_{12} \mathrm{H}_{13}\right)(5)$ \\
\hline 78 & 2861 & 3014.5745 & 2892,67245 & 45.7319 & $v\left(\mathrm{C}_{12} \mathrm{H}_{13}\right)(63) ; \nu\left(\mathrm{C}_{30} \mathrm{H}_{31}\right)(31)$ \\
\hline 79 & 2875 & 3018.0275 & 2895,81595 & 55.9324 & $v\left(\mathrm{C}_{30} \mathrm{H}_{31}\right)(98)$ \\
\hline 80 & - & 3023.8407 & 2901,10723 & 22.2942 & $v\left(\mathrm{C}_{17} \mathrm{H}_{19}\right)(58) ; \nu\left(\mathrm{C}_{17} \mathrm{H}_{18}\right)(29)$ \\
\hline 81 & - & 3026.1436 & 2903,20306 & 16.4278 & $\begin{array}{l}v\left(\mathrm{C}_{17} \mathrm{H}_{19}\right)(32) ; v\left(\mathrm{C}_{14} \mathrm{H}_{2}\right)(29) ; v\left(\mathrm{C}_{17} \mathrm{H}_{18}\right)(15) ; v \\
\left(\mathrm{C}_{30} \mathrm{C}_{31}\right)(8) ; v\left(\mathrm{C}_{21} \mathrm{H}_{2}\right)(6)\end{array}$ \\
\hline 82 & - & 3034.8324 & 2911,10906 & 35.1134 & $\nu\left(\mathrm{C}_{14} \mathrm{H}_{2}\right)(96)$ \\
\hline
\end{tabular}


TABLE 2: Continued.

\begin{tabular}{|c|c|c|c|c|c|}
\hline Number ${ }^{\mathrm{b}}$ & $\begin{array}{c}\text { Observed } \\
\left(\mathrm{cm}^{-1}\right)\end{array}$ & $\begin{array}{l}\text { DFT/6-311+G } \mathrm{G}^{* *} \\
\text { Unscaled }\left(\mathrm{cm}^{-1}\right)\end{array}$ & Scaled $\left(\mathrm{cm}^{-1}\right)$ & $\begin{array}{c}\text { Intensity } \\
\left(\mathrm{km} \cdot \mathrm{mol}^{-1}\right)\end{array}$ & Assignment $(\% \mathrm{PED})^{\mathrm{a}}$ \\
\hline 83 & - & 3041.9158 & 2917,55246 & 16.0910 & $\begin{array}{l}v\left(\mathrm{C}_{27} \mathrm{H}_{2}\right)(38) ; v\left(\mathrm{C}_{9} \mathrm{H}_{2}\right)(20) ; v\left(\mathrm{C}_{14} \mathrm{H}_{2}\right)(19) ; v\left(\mathrm{C}_{21} \mathrm{H}_{2}\right) \\
(6) ; v\left(\mathrm{C}_{24} \mathrm{H}\right)(6)\end{array}$ \\
\hline 84 & - & 3043.1697 & 2918,6929 & 14.5616 & $\begin{array}{l}\nu\left(\mathrm{C}_{27} \mathrm{H}_{2}\right)(47) ; \nu\left(\mathrm{C}_{9} \mathrm{H}_{2}\right)(26) ; \nu\left(\mathrm{C}_{24} \mathrm{H}_{2}\right)(10) ; \nu\left(\mathrm{C}_{14} \mathrm{H}_{2}\right) \\
(7)\end{array}$ \\
\hline 85 & - & 3062.906 & 2936,63657 & 46.5673 & $\begin{array}{l}\nu\left(\mathrm{C}_{21} \mathrm{H}_{2}\right)(55) ; \nu\left(\mathrm{C}_{27} \mathrm{H}_{2}\right)(25) ; \nu\left(\mathrm{C}_{24} \mathrm{H}_{2}\right)(9) ; \nu\left(\mathrm{C}_{12} \mathrm{H}_{13}\right) \\
(5)\end{array}$ \\
\hline 86 & 2931 & 3078.8255 & 2951,10087 & 85.8987 & $\begin{array}{l}\nu\left(\mathrm{C}_{27} \mathrm{H}_{2}\right)(62) ; v\left(\mathrm{C}_{27} \mathrm{H}_{30}\right)(10) ; v 2955,03236\left(\mathrm{C}_{30} \mathrm{H}_{3}\right) \\
(9) ; v\left(\mathrm{C}_{9} \mathrm{H}_{2}\right)(6) ; v\left(\mathrm{C}_{17} \mathrm{H}_{19}\right)(6)\end{array}$ \\
\hline 87 & - & 3083.1541 & 2955,03236 & 45.5349 & $v\left(\mathrm{C}_{30} \mathrm{H}_{31}\right)(99)$ \\
\hline 88 & - & 3084.5244 & 2956,27682 & 35.1235 & $\begin{array}{l}\nu\left(\mathrm{C}_{17} \mathrm{H}_{19}\right)(67) ; \nu\left(\mathrm{C}_{17} \mathrm{C}_{19}\right)(67) ; \nu\left(\mathrm{C}_{17} \mathrm{C}_{18}\right)(17) ; \nu \\
\left(\mathrm{C}_{30} \mathrm{H}_{31}\right)(15)\end{array}$ \\
\hline 89 & - & 3087.9105 & 2959,3517 & 19.5239 & $\begin{array}{l}\nu\left(\mathrm{C}_{9} \mathrm{H}_{2}\right)(63) ; \nu\left(\mathrm{O}_{8} \mathrm{C}_{9}\right)(16) ; \nu-\left(\mathrm{C}_{12} \mathrm{H}_{13}\right)(11) ; \nu\left(\mathrm{C}_{17} \mathrm{C}_{19}\right) \\
(6)\end{array}$ \\
\hline 90 & 2961 & 3093.1423 & 2964,10191 & 56.1433 & $\begin{array}{l}\nu\left(\mathrm{C}_{17} \mathrm{H}_{19}\right)(38) ; \nu\left(\mathrm{C}_{9} \mathrm{H}_{2}\right)(26) ; \nu\left(\mathrm{C}_{17} \mathrm{H}_{20}\right)(12) ; \nu \\
\left(\mathrm{C}_{14} \mathrm{H}_{2}\right)(10) ; \nu\left(\mathrm{C}_{17} \mathrm{H}_{18}\right)(9)\end{array}$ \\
\hline 91 & 3038 & 3142.8663 & 3009,20422 & 5.1952 & $v\left(\mathrm{C}_{1} \mathrm{C}_{4}\right)(45) ; v\left(\mathrm{C}_{1} \mathrm{H}_{2}\right)(38) ; \nu\left(\mathrm{C}_{1} \mathrm{H}_{3}\right)(9)$ \\
\hline 92 & - & 3185.092 & 3047,44188 & 3.2650 & $v\left(\mathrm{C}_{1} \mathrm{H}_{2}\right)(95)$ \\
\hline 93 & 3104 & 3235.9105 & 3093,38372 & 2.3617 & $v\left(\mathrm{C}_{4} \mathrm{H}_{5}\right)(67) ; v\left(\mathrm{C}_{1} \mathrm{H}_{2}\right)(22) ; v\left(\mathrm{C}_{1} \mathrm{H}_{3}\right)(6)$ \\
\hline
\end{tabular}

${ }^{a}$ Assignments and potential energy distribution (PED) (contributing $\geq 5 \%$ ) for vibrational normal mode. Types of vibration: $v$ : stretching; $\delta$ : deformation; o: out-of-plane bending; $\omega$ : wagging; y: twisting; $\rho$ : rocking; $\tau$ : torsion; w: weak; m: medium; sh: shoulder.

${ }^{\mathrm{b}}$ Normal mode number.

$1655 \mathrm{~cm}^{-1}$ for s-cis conformer (Figure 3(b)) and $1647 \mathrm{~cm}^{-1}$ for the s-trans conformer (Figure 3(c)). The band at $1637 \mathrm{~cm}^{-1}$ may be used to definitely indicate that conformer with s-trans arrangement of acrylic moiety is present or not in the liquid 2-ethylhexyl acrylate.

In the region from 1500 to $1200 \mathrm{~cm}^{-1}$, in the experimental spectrum (Figure 3 ), there are four bands of medium intensity located at $1464,1408,1295$, and $1272 \mathrm{~cm}^{-1}$. The bands of weak intensity of account of three are located at 1381,1357 , and $1340 \mathrm{~cm}^{-1}$. The calculated frequencies in this region for the $\mathrm{s}$-cis and s-trans conformations have close energies for each normal mode, and the differences do not exceed $5 \mathrm{~cm}^{-1}$. Based on the assignments reported by Dulce et al. [28] and our PED calculations, we have ascribed the theoretical frequencies $1485 \mathrm{~cm}^{-1}$ (s-cis) and $1484 \mathrm{~cm}^{-1}$ (s-trans) to the experimental band at $1464 \mathrm{~cm}^{-1}$ and they are representing a $\mathrm{C}_{17} \mathrm{H}_{3}$ symmetric bending. The calculated frequencies at 1479,1478 , and $1476 \mathrm{~cm}^{-1}$ may be contributed to the experimental band at $1464 \mathrm{~cm}^{-1}$ due to its relatively large middle width of about $75 \mathrm{~cm}^{-1}$. Mishra et al. [38] have reported the experimental and the theoretical IR spectra of $\gamma$ form of oleic acid, many peaks reported in the region 1370 to $1220 \mathrm{~cm}^{-1}$ for the $\mathrm{CH}_{2}$ carboxyl-sided chain correspond approximately to those we found in our spectrum from 1400 to $1200 \mathrm{~cm}^{-1}$, and all of them may be assigned to $\mathrm{CH}_{2}$ deformation. The assignment is based on the bands intensities; we found a good correlation between the experimental and the theoretical spectra. One should point out that the very intense bands predicted at 1267 and
$1264 \mathrm{~cm}^{-1}$ for s-trans form reproduce well the asymmetric shape of the experimental band at $1272 \mathrm{~cm}^{-1}$. For bands assignment, see Tables 2 and 3.

5.3. The CO Stretching and CH Bending Vibrations (1200$700 \mathrm{~cm}^{-1}$, Figure 4). In this region, in the IR spectrum, there is one very strong band at $1192 \mathrm{~cm}^{-1}$ with weak shoulder at $1160 \mathrm{~cm}^{-1}$ and four medium intensity bands $1057,984,962$, shoulder at 933 and $811 \mathrm{~cm}^{-1}$. The bands at 1116, 1025, 909, $896,852,770$, and $727 \mathrm{~cm}^{-1}$ (shoulder at $748 \mathrm{~cm}^{-1}$ ) are of weak intensity (Figure 4). Based on PEDs and intensities of the calculated frequencies, we correlate the two intense bands at 1192 and $1156 \mathrm{~cm}^{-1}$ for the more stable and the less stable conformers to the band at $1191 \mathrm{~cm}^{-1}$ in the experimental IR spectrum. The medium bands in IR spectrum are well reproduced by the calculated peaks at 1079, 999, 976, 941, and $811 \mathrm{~cm}^{-1}$ for the s-cis conformer and 1067, 993, 940, 911, and $828 \mathrm{~cm}^{-1}$ for the s-trans conformer. They may be ascribed to $\mathrm{CH}_{2}$ rocking, $\mathrm{C}_{1} \mathrm{H}_{2}$ wagging, $\mathrm{CCH}$ rocking $\mathrm{O}_{8} \mathrm{C}_{9}$, and $\mathrm{C}_{1} \mathrm{H}_{2}$ twisting. The peaks predicted by DFT at 728 and $783 \mathrm{~cm}^{-1}$ in s-cis form and the peaks at 726 and $782 \mathrm{~cm}^{-1}$ in the s-trans form may be correlated to the bands at 727 and $770 \mathrm{~cm}^{-1}$ and they are assigned to the $\mathrm{CH}_{2}$ deformations of the alkyl sides of the title molecule.

In the IR spectra of 2-ethylhexyl acrylate, reported in this work, there are a few bands with weak to very weak intensity which do not appear in the IR spectra of methyl acrylate reported by Dulce et al. [28] and George et al. [39]. These bands appear at 1157, 1120,1052, and $1022 \mathrm{~cm}^{-1}$, and they 
TABle 3: Frequencies and PEDs of s-trans 2-ethylhexyl acrylate.

\begin{tabular}{|c|c|c|c|c|c|}
\hline Number ${ }^{\mathrm{b}}$ & $\begin{array}{c}\text { Observed } \\
\left(\mathrm{cm}^{-1}\right)\end{array}$ & $\begin{array}{l}\text { DFT/6-311+G } \mathrm{G}^{* *} \\
\text { Unscaled }\left(\mathrm{cm}^{-1}\right)\end{array}$ & Scaled $\left(\mathrm{cm}^{-1}\right)$ & $\begin{array}{c}\text { Intensity } \\
\left(\mathrm{km} \cdot \mathrm{mol}^{-1}\right)\end{array}$ & Assignment $(\% \mathrm{PED})^{\mathrm{a}}$ \\
\hline 1 & - & 29.5569 & 29,79981 & 1.3888 & $\tau\left(\mathrm{C}_{21} \mathrm{C}_{24}\right)(95)$ \\
\hline 2 & - & 31.2922 & 31,54848 & 1.2915 & $\tau\left(\mathrm{C}_{21} \mathrm{C}_{24}\right)(100)$ \\
\hline 3 & - & 45.3002 & 45,66086 & 0.0227 & $\tau\left(\mathrm{C}_{21} \mathrm{C}_{24}\right)(91) ; \tau\left(\mathrm{O}_{8} \mathrm{C}_{9}\right)(6)$ \\
\hline 4 & - & 69.075 & 69,59818 & 0.0113 & $\tau\left(\mathrm{C}_{21} \mathrm{C}_{24}\right)(98)$ \\
\hline 5 & - & 91.6561 & 92,31657 & 0.7304 & $\rho\left(\mathrm{C}_{14} \mathrm{H}_{2}\right)(64) ; \tau\left(\mathrm{C}_{9} \mathrm{C}_{12}\right)(19) ; \tau\left(\mathrm{C}_{24} \mathrm{C}_{21}\right)(15)$ \\
\hline 6 & - & 106.795 & 107,53821 & 0.0788 & $\tau\left(\mathrm{C}_{12} \mathrm{C}_{14}\right)(92)$ \\
\hline 7 & - & 116.406 & 117,19786 & 0.4606 & $\tau\left(\mathrm{O}_{8} \mathrm{C}_{9}\right)(90) ; \tau\left(\mathrm{C}_{21} \mathrm{C}_{24}\right)(7)$ \\
\hline 8 & - & 128.002 & 128,84855 & 0.7652 & $\tau\left(\mathrm{C}_{21} \mathrm{C}_{24}\right)(99)$ \\
\hline 9 & - & 137.7311 & 138,62015 & 0.0737 & $\tau\left(\mathrm{C}_{24} \mathrm{C}_{21}\right)(94)$ \\
\hline 10 & - & 188.6176 & 189,67867 & 2.4795 & $\tau\left(\mathrm{C}_{24} \mathrm{C}_{21}\right)(53) ; \mathrm{O}\left(\mathrm{O}_{8} \mathrm{C}_{9}\right)$ \\
\hline 11 & - & 196.4898 & 197,56995 & 2.2280 & $\rho\left(\mathrm{C}_{9} \mathrm{H}_{2}\right)(82) ; \tau\left(\mathrm{C}_{9} \mathrm{C}_{12}\right)(13)$ \\
\hline 12 & - & 234.6 & 235,74391 & 1.7535 & $\rho\left(\mathrm{C}_{30} \mathrm{H}_{3}\right)(90) ; \tau\left(\mathrm{C}_{21} \mathrm{C}_{24}\right)(7)$ \\
\hline 13 & - & 249.5865 & 250,74252 & 3.2507 & $\rho\left(\mathrm{C}_{30} \mathrm{H}_{3}\right)(78) ; \tau\left(\mathrm{C}_{12} \mathrm{C}_{21}\right)$ \\
\hline 14 & - & 262.3636 & 263,52416 & 1.2259 & $\rho\left(\mathrm{C}_{9} \mathrm{H}_{2}\right)(72) ; \rho\left(\mathrm{C}_{17} \mathrm{H}_{3}\right)(22)$ \\
\hline 15 & - & 274.1636 & 275,32362 & 0.5126 & $\tau\left(\mathrm{C}_{12} \mathrm{C}_{21}\right)(96)$ \\
\hline 16 & - & 335.2283 & 336,31302 & 0.4554 & $\delta\left(\mathrm{C}_{21} \mathrm{C}_{12} \mathrm{C}_{14}\right)(93)$ \\
\hline 17 & - & 354.6136 & 355,649 & 0.1655 & $\delta\left(\mathrm{C}_{21} \mathrm{C}_{12} \mathrm{C}_{24}\right)(95)$ \\
\hline 18 & - & 406.411 & 407,25451 & 2.5402 & $\delta\left(\mathrm{C}_{9} \mathrm{C}_{12} \mathrm{C}_{14}\right)(92)$ \\
\hline 19 & - & 471.3032 & 471,78287 & 1.0320 & $\rho\left(\mathrm{C}_{9} \mathrm{C}_{12} \mathrm{C}_{14}\right)(70) ; \delta\left(\mathrm{C}_{9} \mathrm{C}_{12} \mathrm{C}_{21}\right)(12) ; \rho\left(\mathrm{C}_{14} \mathrm{H}_{2}\right)(11)$ \\
\hline 20 & - & 509.8943 & 510,09251 & 0.4733 & $\mathrm{y}\left(\mathrm{C}_{1} \mathrm{H}_{2}\right)(86) ; \mathrm{O}\left(\mathrm{C}_{6} \mathrm{O}_{8}\right)(12)$ \\
\hline 21 & - & 514.0157 & 514,18098 & 7.3067 & $\begin{array}{l}\rho\left(\mathrm{C}_{12} \mathrm{C}_{14} \mathrm{C}_{17}\right)(38) ; \rho\left(\mathrm{C}_{9} \mathrm{C}_{12} \mathrm{C}_{21}\right)(15) ; \rho\left(\mathrm{C}_{12} \mathrm{C}_{21}\right)(14) ; \\
\rho\left(\mathrm{C}_{14} \mathrm{C}_{12} \mathrm{H}_{13}\right)(13) ; \rho\left(\mathrm{C}_{1} \mathrm{H}_{2}\right)(8) ; \delta\left(\mathrm{C}_{12} \mathrm{C}_{21} \mathrm{C}_{24}\right)(7) ;\end{array}$ \\
\hline 22 & - & 550.859 & 550,70531 & 2.2808 & $\rho\left(\mathrm{C}_{9} \mathrm{C}_{12} \mathrm{C}_{14}\right)(78) ; \rho\left(\mathrm{C}_{6} \mathrm{O}_{8} \mathrm{C}_{9}\right)(9) ; \rho\left(\mathrm{C}_{1} \mathrm{H}_{2}\right)(6)$ \\
\hline 23 & - & 636.0175 & 634,9572 & 1.9146 & $\rho\left(\mathrm{O}_{8} \mathrm{C}_{9} \mathrm{C}_{12}\right)(97)$ \\
\hline 24 & $727(\mathrm{~m})$ & 728.4338 & 726,12214 & 4.3677 & $\rho\left(\mathrm{C}_{9} \mathrm{C}_{12} \mathrm{C}_{21}\right)(92)$ \\
\hline 25 & - & 778.027 & 774,92902 & 0.6843 & $\rho\left(\mathrm{C}_{9} \mathrm{C}_{12} \mathrm{C}_{21}\right)(98)$ \\
\hline 26 & $770(\mathrm{~m})$ & 785.397 & 782,17532 & 1.8732 & $\rho\left(\mathrm{C}_{21} \mathrm{H}_{2}\right)(59) ; \rho\left(\mathrm{C}_{14} \mathrm{H}_{2}\right)(36) ;$ \\
\hline 27 & $811(\mathrm{~m})$ & 832.1364 & 828,08904 & 21.5147 & $\mathrm{y}\left(\mathrm{C}_{1} \mathrm{H}_{2}\right)(94) ; \rho\left(\mathrm{C}_{9} \mathrm{H}_{2}\right)(5)$ \\
\hline 28 & $852(\mathrm{w})$ & 850.1904 & 845,80503 & 3.4731 & $\begin{array}{l}\rho\left(\mathrm{C}_{9} \mathrm{C}_{12} \mathrm{C}_{21}\right)(47) ; \rho\left(\mathrm{C}_{9} \mathrm{H}_{2}\right)(27) ; \rho\left(\mathrm{C}_{1} \mathrm{H}_{2}\right)(15) ; \nu \\
\left(\mathrm{C}_{14} \mathrm{C}_{17}\right)(5) ;\end{array}$ \\
\hline 29 & $896(w)$ & 901.7561 & 896,3468 & 0.9206 & $\rho\left(\mathrm{C}_{9} \mathrm{C}_{12} \mathrm{C}_{21}\right)(73) ; \rho\left(\mathrm{C}_{21} \mathrm{C}_{24} \mathrm{C}_{27}\right)(14)$ \\
\hline 30 & $909(\mathrm{w})$ & 906.5632 & 901,05403 & 1.3451 & $\rho\left(\mathrm{C}_{9} \mathrm{C}_{12} \mathrm{C}_{21}\right)(46) ; \rho\left(\mathrm{C}_{9} \mathrm{H}_{2}\right)(35) ; \rho\left(\mathrm{C}_{12} \mathrm{C}_{14} \mathrm{C}_{17}\right)(9) ;$ \\
\hline 31 & $933(\mathrm{sh})$ & 917.0283 & 911,29911 & 7.6343 & $\rho\left(\mathrm{O}_{7} \mathrm{C}_{6} \mathrm{O}_{8}\right)(86) ; \rho\left(\mathrm{C}_{9} \mathrm{H}_{2}\right)(10)$ \\
\hline 32 & $962(\mathrm{~m})$ & 947.0255 & 940,64585 & 6.0649 & $\rho\left(\mathrm{C}_{6} \mathrm{O}_{8} \mathrm{C}_{9}\right)(99)$ \\
\hline 33 & - & 972.6775 & 965,71834 & 7.0484 & $\rho\left(\mathrm{C}_{6} \mathrm{O}_{8} \mathrm{C}_{9}\right)(99)$ \\
\hline 34 & $984(\mathrm{~m})$ & 1001.2212 & 993,59199 & 34.4957 & $\begin{array}{l}\omega\left(\mathrm{C}_{1} \mathrm{H}_{2}\right)(52) ; \nu\left(\mathrm{C}_{1} \mathrm{C}_{4}\right)(15) ; \delta\left(\mathrm{C}_{4} \mathrm{C}_{6} \mathrm{O}_{8}\right)(15) ; \nu\left(\mathrm{C}_{12} \mathrm{C}_{21}\right) \\
(15)\end{array}$ \\
\hline 35 & $1015(\mathrm{sh})$ & 1017.1676 & 1009,15249 & 7.0006 & $\nu\left(\mathrm{C}_{12} \mathrm{C}_{21}\right)(92)$ \\
\hline 36 & - & 1021.9635 & 1013,83071 & 3.5045 & $\nu\left(\mathrm{C}_{21} \mathrm{C}_{24}\right)(85) ; \delta\left(\mathrm{C}_{9} \mathrm{C}_{12} \mathrm{O}_{14}\right)(9)$ \\
\hline 37 & $1025(\mathrm{sh})$ & 1029.9052 & 1021,57589 & 18.5130 & $\mathrm{y}\left(\mathrm{C}_{1} \mathrm{H}_{2}\right)(97)$ \\
\hline 38 & - & 1040.755 & 1032,15388 & 0.3554 & $v\left(\mathrm{C}_{21} \mathrm{C}_{24}\right)(70) ; \nu\left(\mathrm{C}_{9} \mathrm{C}_{12}\right)(14) ; \mathrm{y}\left(\mathrm{C}_{21} \mathrm{H}_{2}\right)(5)$ \\
\hline 39 & - & 1060.8287 & 1051,71458 & 5.5936 & $\mathrm{y}\left(\mathrm{C}_{21} \mathrm{H}_{2}\right)(58) ; \rho\left(\mathrm{C}_{14} \mathrm{C}_{12} \mathrm{H}_{13}\right)(29) ; \rho\left(\mathrm{C}_{14} \mathrm{H}_{2}\right)(7)$ \\
\hline 40 & - & 1064.0573 & 1054,85945 & 18.8097 & $\nu\left(\mathrm{C}_{21} \mathrm{C}_{24}\right)(88) ; \nu\left(\mathrm{C}_{14} \mathrm{C}_{17}\right)(8)$ \\
\hline 41 & $1057(\mathrm{~m})$ & 1076.7989 & 1067,26727 & 192.6571 & $v\left(\mathrm{C}_{9} \mathrm{O}_{8}\right)(82) ; \nu\left(\mathrm{C}_{14} \mathrm{C}_{17}\right)(13)$ \\
\hline 42 & $1060(\mathrm{sh})$ & 1089.5069 & 1079,6371 & 4.6500 & $\rho\left(\mathrm{C}_{9} \mathrm{H}_{2}\right)(40) ; \delta\left(\mathrm{C}_{9} \mathrm{C}_{12} \mathrm{C}_{21}\right)(30) ; \omega\left(\mathrm{C}_{27} \mathrm{H}_{2}\right)(25)$ \\
\hline 43 & $1116(w)$ & 1150.6075 & 1139,03825 & 1.4631 & $\rho\left(\mathrm{C}_{9} \mathrm{C}_{12} \mathrm{H}_{13}\right)(51) ; \delta\left(\mathrm{C}_{9} \mathrm{C}_{12} \mathrm{C}_{21}\right)$ \\
\hline 44 & 1191 & 1168.2621 & 1156,17915 & 3.2253 & $\rho\left(\mathrm{C}_{14} \mathrm{C}_{12} \mathrm{H}_{13}\right)(72) ; \omega\left(\mathrm{C}_{21} \mathrm{H}_{2}\right)(16) ; \rho\left(\mathrm{O}_{8} \mathrm{C}_{9} \mathrm{C}_{12}\right)(9)$ \\
\hline 45 & - & 1185.0265 & 1172,44634 & 0.1695 & $\delta\left(\mathrm{C}_{9} \mathrm{C}_{12} \mathrm{C}_{14}\right)(94)$ \\
\hline
\end{tabular}


TABle 3: Continued.

\begin{tabular}{|c|c|c|c|c|c|}
\hline Number ${ }^{\mathrm{b}}$ & $\begin{array}{c}\text { Observed } \\
\left(\mathrm{cm}^{-1}\right)\end{array}$ & $\begin{array}{l}\text { DFT/6-311+G } \mathrm{G}^{* *} \\
\text { Unscaled }\left(\mathrm{cm}^{-1}\right)\end{array}$ & Scaled $\left(\mathrm{cm}^{-1}\right)$ & $\begin{array}{c}\text { Intensity } \\
\left(\mathrm{km} \cdot \mathrm{mol}^{-1}\right)\end{array}$ & Assignment $(\% \mathrm{PED})^{\mathrm{a}}$ \\
\hline 46 & - & 1242.923 & 1228,55525 & 1.7298 & $\mathrm{y}\left(\mathrm{C}_{21} \mathrm{H}_{2}\right)(89)$ \\
\hline 47 & - & 1258.238 & 1243,37912 & 1.1546 & $\rho\left(\mathrm{C}_{9} \mathrm{C}_{12} \mathrm{C}_{21}\right)(35) ; \omega\left(\mathrm{C}_{21} \mathrm{H}_{2}\right)(35) ; \omega\left(\mathrm{C}_{9} \mathrm{H}_{2}\right)(27)$ \\
\hline 48 & - & 1280.1017 & 1264,52842 & 77.9146 & $\omega\left(\mathrm{C}_{21} \mathrm{H}_{2}\right)(47) ; \rho\left(\mathrm{C}_{9} \mathrm{C}_{12} \mathrm{C}_{21}\right)(37) ; \rho\left(\mathrm{C}_{9} \mathrm{H}_{2}\right)(27) ;$ \\
\hline 49 & 1272 & 1283.202 & 1267,52616 & 351.6365 & $\omega\left(\mathrm{C}_{21} \mathrm{H}_{2}\right)(60) ; \mathrm{y}\left(\mathrm{C}_{14} \mathrm{H}_{2}\right)(27) ; \mathrm{y}\left(\mathrm{C}_{9} \mathrm{H}_{2}\right)(10)$ \\
\hline 50 & - & 1299.1286 & 1282,92094 & 4.4292 & $\mathrm{y}\left(\mathrm{C}_{9} \mathrm{H}_{2}\right)(72) ; \mathrm{y}\left(\mathrm{C}_{21} \mathrm{H}_{2}\right)(26)$ \\
\hline 51 & 1295 & 1311.7811 & 1295,14505 & 7.2842 & $\mathrm{y}\left(\mathrm{C}_{14} \mathrm{H}_{2}\right)(99)$ \\
\hline 52 & - & 1318.75 & 1301,87577 & 6.8204 & $\mathrm{y}\left(\mathrm{C}_{14} \mathrm{H}_{2}\right)(100)$ \\
\hline 53 & - & 1326.4691 & 1309,3292 & 0.1479 & $\mathrm{y}\left(\mathrm{C}_{21} \mathrm{H}_{2}\right)(97)$ \\
\hline 54 & - & 1336.2199 & 1318,74163 & 0.4090 & $\mathrm{y}\left(\mathrm{C}_{24} \mathrm{H}_{2}\right)(94) ; \mathrm{y}\left(\mathrm{C}_{21} \mathrm{H}_{2}\right)(5)$ \\
\hline 55 & 1340 & 1359.997 & 1341,68063 & 1.0793 & $\omega\left(\mathrm{C}_{21} \mathrm{H}_{2}\right)(86) ; \rho\left(\mathrm{C}_{21} \mathrm{C}_{12} \mathrm{C}_{14}\right)(7) ; \omega\left(\mathrm{C}_{9} \mathrm{H}_{2}\right)(5)$ \\
\hline 56 & - & 1380.0145 & 1360,97825 & 0.4801 & $\rho\left(\mathrm{C}_{9} \mathrm{C}_{12} \mathrm{C}_{21}\right)(43) ; \delta\left(\mathrm{C}_{9} \mathrm{H}_{12}\right)(28) ; \omega\left(\mathrm{C}_{21} \mathrm{H}_{2}\right)(25)$ \\
\hline 57 & 1357 & 1393.1134 & 1373,59902 & 2.8872 & $\begin{array}{l}\omega\left(\mathrm{C}_{9} \mathrm{H}_{2}\right)(50) ; \omega\left(\mathrm{C}_{14} \mathrm{H}_{2}\right)(23) ; \delta\left(\mathrm{C}_{21} \mathrm{C}_{12} \mathrm{H}_{13}\right)(12) ; \omega \\
\left(\mathrm{C}_{21} \mathrm{H}_{2}\right)(86)\end{array}$ \\
\hline 58 & - & 1404.3849 & 1384,45461 & 0.9476 & $\omega\left(\mathrm{C}_{21} \mathrm{H}_{2}\right)(86) ; \omega\left(\mathrm{C}_{9} \mathrm{H}_{2}\right)(12)$ \\
\hline 59 & - & 1411.9509 & 1391,73911 & 0.8054 & $\omega\left(\mathrm{C}_{9} \mathrm{H}_{2}\right)(85) ; \omega\left(\mathrm{C}_{21} \mathrm{H}_{2}\right)(14)$ \\
\hline 60 & 1381 & 1414.5333 & 1394,225 & 5.0233 & $\omega\left(\mathrm{C}_{21} \mathrm{H}_{2}\right)(54) ; \omega\left(\mathrm{C}_{9} \mathrm{H}_{2}\right)(45)$ \\
\hline 61 & - & 1417.3104 & 1396,89807 & 0.8100 & $\omega\left(\mathrm{C}_{21} \mathrm{H}_{2}\right)(89) ; \omega\left(\mathrm{C}_{9} \mathrm{H}_{2}\right)(8)$ \\
\hline 62 & 1408 & 1441.4398 & 1420,11302 & 30.5726 & $\omega\left(\mathrm{C}_{9} \mathrm{H}_{2}\right)(93) ; \omega\left(\mathrm{C}_{21} \mathrm{H}_{2}\right)(5)$ \\
\hline 63 & - & 1483.1028 & 1460,15241 & 2.0326 & $\begin{array}{l}\delta\left(\mathrm{C}_{9} \mathrm{H}_{2}\right)(38) ; \delta\left(\mathrm{C}_{21} \mathrm{C}_{12} \mathrm{H}_{13}\right)(18) ; \delta\left(\mathrm{C}_{24} \mathrm{C}_{21} \mathrm{C}_{12}\right)(15) ; \delta \\
\left(\mathrm{C}_{14} \mathrm{H}_{2}\right)(13) ; \delta\left(\mathrm{C}_{24} \mathrm{H}_{2}\right)(7) ; \delta\left(\mathrm{C}_{21} \mathrm{H}_{2}\right)(6)\end{array}$ \\
\hline 64 & - & 1487.6468 & 1464,51591 & 0.4300 & $\begin{array}{l}\delta\left(\mathrm{C}_{21} \mathrm{C}_{12} \mathrm{H}_{13}\right)(33) ; \delta\left(\mathrm{C}_{27} \mathrm{H}_{2}\right)(28) ; \delta\left(\mathrm{C}_{24} \mathrm{H}_{2}\right)(20) ; \delta \\
\left(\mathrm{C}_{21} \mathrm{H}_{2}\right)(10)\end{array}$ \\
\hline 65 & - & 1491.6884 & 1468,3964 & 1.2432 & $\delta\left(\mathrm{C}_{21} \mathrm{H}_{2}\right)(54) ; \delta\left(\mathrm{C}_{9} \mathrm{H}_{2}\right)(21) ; \delta\left(\mathrm{C}_{27} \mathrm{H}_{2}\right)(11)$ \\
\hline 66 & - & 1500.0764 & 1476,44833 & 2.4692 & $\begin{array}{l}\delta\left(\mathrm{C}_{24} \mathrm{H}_{2}\right)(32) ; \delta\left(\mathrm{C}_{21} \mathrm{C}_{12} \mathrm{H}_{13}\right)(24) ; \delta\left(\mathrm{C}_{27} \mathrm{H}_{2}\right)(8) ; \delta \\
\left(\mathrm{C}_{30} \mathrm{H}_{3}\right)(8) ; \delta\left(\mathrm{C}_{21} \mathrm{H}_{2}\right)(6)\end{array}$ \\
\hline 67 & - & 1500.718 & 1477,06413 & 7.0795 & $\delta\left(\mathrm{C}_{30} \mathrm{H}_{3}\right)(75) ; \delta\left(\mathrm{C}_{9} \mathrm{H}_{2}\right)(11) ; \delta\left(\mathrm{C}_{27} \mathrm{H}_{2}\right)(6)$ \\
\hline 68 & - & 1502.5266 & 1478,79993 & 8.9950 & $\delta\left(\mathrm{C}_{9} \mathrm{H}_{2}\right)(74) ; \delta\left(\mathrm{C}_{17} \mathrm{H}_{3}\right)(21)$ \\
\hline 69 & 1464 & 1508.1671 & 1484,21269 & 9.8400 & $\begin{array}{l}\delta\left(\mathrm{C}_{27} \mathrm{H}_{2}\right)(26) ; \delta\left(\mathrm{C}_{30} \mathrm{H}_{3}\right)(22) ; \delta\left(\mathrm{C}_{21} \mathrm{H}_{2}\right)(14) ; \delta \\
\left(\mathrm{C}_{17} \mathrm{H}_{3}\right)(14) ; \delta\left(\mathrm{C}_{24} \mathrm{H}_{2}\right)(11)\end{array}$ \\
\hline 70 & - & 1511.6225 & 1487,52807 & 10.5533 & $\begin{array}{l}\delta\left(\mathrm{C}_{21} \mathrm{H}_{2}\right)(73) ; \delta\left(\mathrm{C}_{21} \mathrm{C}_{12} \mathrm{H}_{13}\right)(6) ; \delta\left(\mathrm{C}_{24} \mathrm{H}_{2}\right)(5) ; \delta \\
\left(\mathrm{C}_{17} \mathrm{H}_{3}\right)(5)\end{array}$ \\
\hline 71 & - & 1514.069 & 1489,8752 & 5.6625 & $\begin{array}{l}\delta\left(\mathrm{C}_{24} \mathrm{H}_{2}\right)(30) ; \delta\left(\mathrm{C}_{21} \mathrm{H}_{2}\right)(29) ; \delta\left(\mathrm{C}_{21} \mathrm{C}_{24} \mathrm{C}_{27}\right)(20) ; \delta \\
\left(\mathrm{C}_{27} \mathrm{H}_{2}\right)(9)\end{array}$ \\
\hline 72 & 1619 & 1678.7098 & 1647,38009 & 28.7096 & $\begin{array}{l}\nu\left(\mathrm{C}_{1} \mathrm{C}_{4}\right)(52) ; \nu\left(\mathrm{C}_{4} \mathrm{C}_{6}\right)(22) ; \delta\left(\mathrm{C}_{9} \mathrm{C}_{12} \mathrm{H}_{13}\right)(11) ; \delta \\
\left(\mathrm{C}_{21} \mathrm{H}_{2}\right)(8)\end{array}$ \\
\hline 73 & 1727 & 1766.8533 & 1731,34006 & 315.5882 & $\begin{array}{l}\delta\left(\mathrm{C}_{9} \mathrm{C}_{12} \mathrm{C}_{21}\right)(63) ; \nu\left(\mathrm{C}_{4} \mathrm{C}_{6}\right)(16) ; \delta\left(\mathrm{C}_{21} \mathrm{H}_{2}\right)(6) ; \nu \\
\left(\mathrm{C}_{6} \mathrm{O}_{7}\right)(6)\end{array}$ \\
\hline 74 & - & 2995.932 & 2875,69419 & 2.0873 & $v\left(\mathrm{C}_{24} \mathrm{H}_{2}\right)(35) ; v\left(\mathrm{C}_{14} \mathrm{H}_{2}\right)(34) ; v\left(\mathrm{C}_{21} \mathrm{H}_{2}\right)(25)$ \\
\hline 75 & - & 2998.467 & 2878,00355 & 0.6759 & $\begin{array}{l}\nu\left(\mathrm{C}_{24} \mathrm{H}_{2}\right)(40) ; \nu\left(\mathrm{C}_{12} \mathrm{H}_{13}\right)(29) ; \nu\left(\mathrm{C}_{21} \mathrm{H}_{2}\right)(12) ; \nu \\
\left(\mathrm{C}_{14} \mathrm{H}_{2}\right)(10)\end{array}$ \\
\hline 76 & - & 3001.649 & 2880,90203 & 13.5187 & $v\left(\mathrm{C}_{21} \mathrm{H}_{2}\right)(92)$ \\
\hline 77 & - & 3005.3152 & 2884,24116 & 13.2101 & $v\left(\mathrm{C}_{24} \mathrm{H}_{2}\right)(87) ; \nu\left(\mathrm{C}_{24} \mathrm{H}_{2}\right)(8)$ \\
\hline 78 & 2861 & 3013.385 & 2891,58948 & 58.5964 & $v\left(\mathrm{C}_{21} \mathrm{H}_{2}\right)(45) ; v\left(\mathrm{C}_{30} \mathrm{C}_{32}\right)(36) ; \nu\left(\mathrm{C}_{12} \mathrm{H}_{13}\right)(12)$ \\
\hline 79 & 2875 & 3017.8939 & 2895,69433 & 47.2871 & $v\left(\mathrm{C}_{30} \mathrm{C}_{32}\right)(96)$ \\
\hline 80 & - & 3023.6248 & 2900,91073 & 21.1397 & $v\left(\mathrm{C}_{14} \mathrm{H}_{12}\right)(46) ; v\left(\mathrm{C}_{17} \mathrm{H}_{19}\right)(32) ; v\left(\mathrm{C}_{17} \mathrm{H}_{18}\right)(17)$ \\
\hline 81 & - & 3026.0401 & 2903,10887 & 15.9088 & $\begin{array}{l}\nu\left(\mathrm{C}_{12} \mathrm{H}_{13}\right)(30) ; \nu\left(\mathrm{C}_{17} \mathrm{H}_{19}\right)(27) ; \nu\left(\mathrm{C}_{17} \mathrm{H}_{18}\right)(13) ; \nu \\
\left(\mathrm{C}_{30} \mathrm{C}_{32}\right)(12)\end{array}$ \\
\hline 82 & - & 3034.55 & 2910,85214 & 32.9164 & $v\left(\mathrm{C}_{21} \mathrm{H}_{2}\right)(66) ; \nu\left(\mathrm{C}_{14} \mathrm{H}_{2}\right)(28)$ \\
\hline 83 & - & 3040.4085 & 2916,18149 & 4.0314 & $v\left(\mathrm{C}_{21} \mathrm{H}_{2}\right)(55) ; v\left(\mathrm{C}_{30} \mathrm{H}_{32}\right)(27) ; v\left(\mathrm{C}_{12} \mathrm{H}_{13}\right)(7)$ \\
\hline 84 & - & 3043.7899 & 2919,25696 & 26.3738 & $v\left(\mathrm{C}_{21} \mathrm{H}_{2}\right)(98)$ \\
\hline 85 & - & 3060.9167 & 2934,82854 & 50.3404 & $v\left(\mathrm{C}_{21} \mathrm{H}_{2}\right)(85) ; v\left(\mathrm{C}_{30} \mathrm{C}_{32}\right)(12)$ \\
\hline 86 & 2931 & 3078.6333 & 2950,92629 & 82.4131 & $v\left(\mathrm{C}_{30} \mathrm{C}_{32}\right)(95)$ \\
\hline
\end{tabular}


TABLE 3: Continued.

\begin{tabular}{|c|c|c|c|c|c|}
\hline Number ${ }^{b}$ & $\begin{array}{l}\text { Observed } \\
\left(\mathrm{cm}^{-1}\right)\end{array}$ & $\begin{array}{l}\text { DFT/6-311+G } \mathrm{G}^{* *} \\
\text { Unscaled }\left(\mathrm{cm}^{-1}\right)\end{array}$ & Scaled $\left(\mathrm{cm}^{-1}\right)$ & $\begin{array}{c}\text { Intensity } \\
\left(\mathrm{km} \cdot \mathrm{mol}^{-1}\right)\end{array}$ & Assignment $(\% \mathrm{PED})^{\mathrm{a}}$ \\
\hline 87 & - & 3083.3975 & 2955,25341 & 44.1245 & $\nu\left(\mathrm{C}_{30} \mathrm{C}_{32}\right)(93)$ \\
\hline 88 & - & 3084.2781 & 2956,05315 & 36.9537 & $\nu\left(\mathrm{C}_{17} \mathrm{H}_{19}\right)(67) ; \nu\left(\mathrm{C}_{17} \mathrm{H}_{18}\right)(18) ; \nu\left(\mathrm{C}_{21} \mathrm{H}_{2}\right)(9)$ \\
\hline 89 & - & 3088.9498 & 2960,29541 & 15.1201 & $\begin{array}{l}\nu\left(\mathrm{C}_{21} \mathrm{H}_{2}\right)(52) ; \nu\left(\mathrm{C}_{9} \mathrm{H}_{2}\right)(14) ; \nu\left(\mathrm{C}_{12} \mathrm{H}_{13}\right)(14) ; \nu\left(\mathrm{C}_{14} \mathrm{H}_{2}\right) \\
(8) ; \nu\left(\mathrm{C}_{17} \mathrm{H}_{19}\right)(7)\end{array}$ \\
\hline 90 & 2961 & 3093.0704 & 2964,03663 & 59.5099 & $\begin{array}{l}\nu\left(\mathrm{C}_{17} \mathrm{H}_{19}\right)(36) ; v\left(\mathrm{C}_{14} \mathrm{H}_{2}\right)(28) ; v\left(\mathrm{C}_{17} \mathrm{H}_{20}\right)(10) ; v \\
\left(\mathrm{C}_{21} \mathrm{H}_{2}\right)(10) ; v\left(\mathrm{C}_{17} \mathrm{H}_{18}\right)(8) ; v\left(\mathrm{C}_{9} \mathrm{H}_{2}\right)(6)\end{array}$ \\
\hline 91 & 3038 & 3146.2567 & 3012,27655 & 5.2018 & $\nu\left(\mathrm{C}_{1} \mathrm{C}_{4}\right)(50) ; \nu\left(\mathrm{C}_{1} \mathrm{H}_{2}\right)(31) ; \nu\left(\mathrm{C}_{1} \mathrm{H}_{3}\right)(10)$ \\
\hline 92 & 3069 & 3185.7999 & 3048,08243 & 3.4136 & $v\left(\mathrm{C}_{4} \mathrm{H}_{5}\right)(57) ; \nu\left(\mathrm{C}_{4} \mathrm{C}_{6}\right)(23) ; \nu\left(\mathrm{C}_{1} \mathrm{C}_{4}\right)(14)$ \\
\hline 93 & 3104 & 3237.707 & 3095,00628 & 4.4771 & $v\left(\mathrm{C}_{1} \mathrm{H}_{2}\right)(70) ; v\left(\mathrm{C}_{1} \mathrm{H}_{3}\right)(11) ; v\left(\mathrm{C}_{4} \mathrm{H}_{5}\right)(7)$ \\
\hline
\end{tabular}

${ }^{a}$ Assignments and potential energy distribution (PED) (contributing $\geq 5 \%$ ) for vibrational normal mode. Types of vibration: $v$ : stretching; $\delta$ : deformation; o: out-of-plane bending; $\omega$ : wagging; y: twisting; $\rho$ : rocking; $\tau$ : torsion; w: weak; m: medium; sh: shoulder.

${ }^{\mathrm{b}}$ Normal mode number.

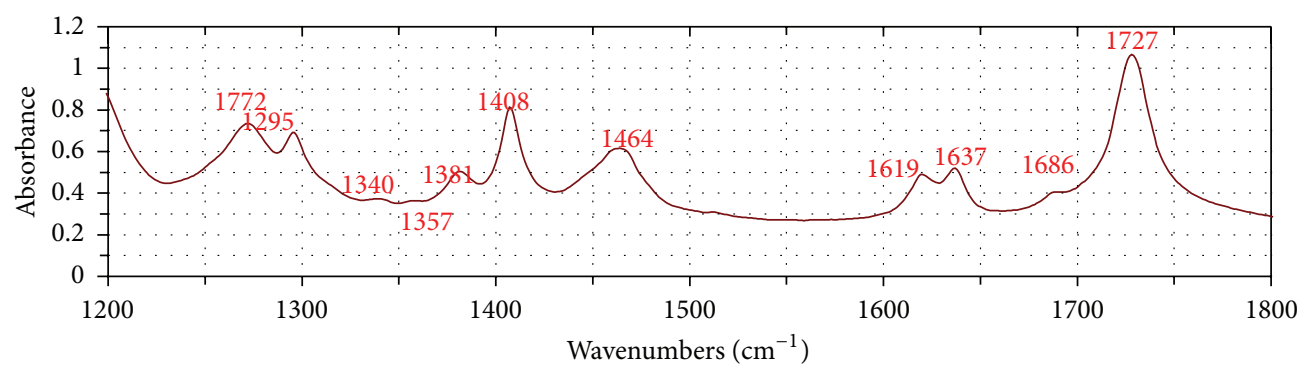

(a)

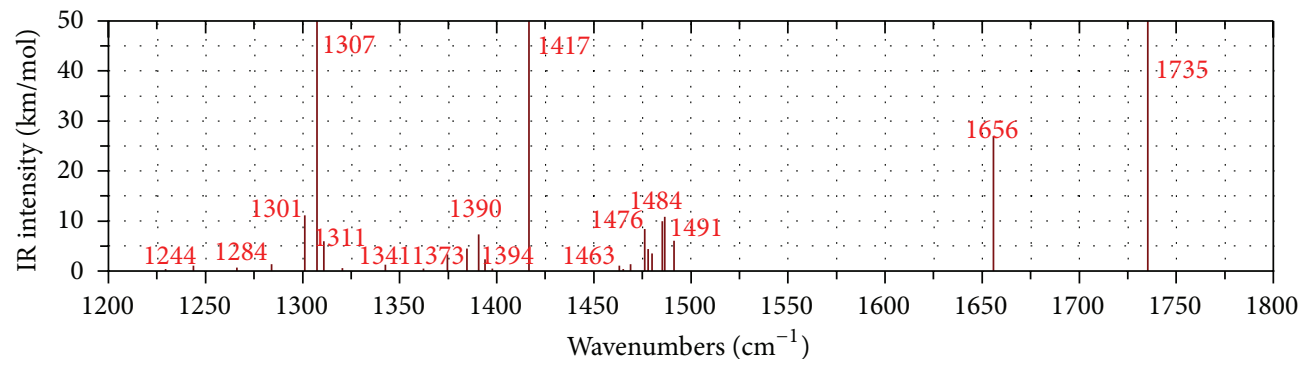

(b)

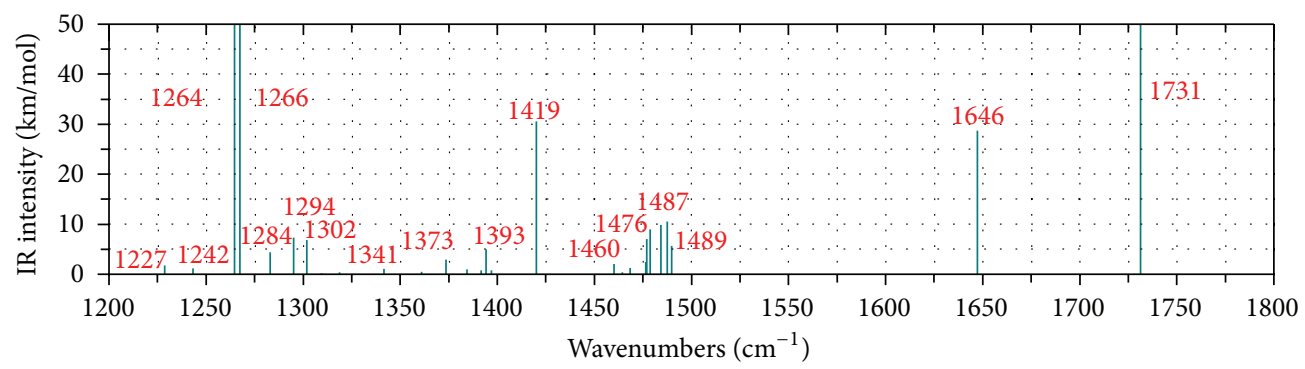

(c)

FIgURE 3: Comparison of the experimental IR (a) $\mathrm{C}=\mathrm{O}$ and $\mathrm{C}=\mathrm{C}$ stretching and $\mathrm{CH}$ bending region (1800-1200 $\mathrm{cm}^{-1}$ ) with the appropriate theoretical B3LYP/6-311+G $\mathrm{G}^{* *}$ spectra ((b) and (c)) for s-cis and s-trans 2-ethylhexyl acrylate, respectively.

are well correlated to the calculated peaks in DFT for both conformers; see Tables 2 and 3.

The shoulders in the infrared spectrum at $1025,1015 \mathrm{~cm}^{-1}$ of weak to medium intensities may be correlated to the bands $1037,1015 \mathrm{~cm}^{-1}$ for s-cis and $1015,988 \mathrm{~cm}^{-1}$ for the s-trans conformer. These bands are ascribed to the $\mathrm{CH}$ out-of-plane bending modes of the vinyl group.

The remaining bands of weak intensity in the experimental IR spectrum are 909,896 , and $852 \mathrm{~cm}^{-1}$. These bands are close to the ones at 911,996 , and $847 \mathrm{~cm}^{-1}$ for the s-cis 


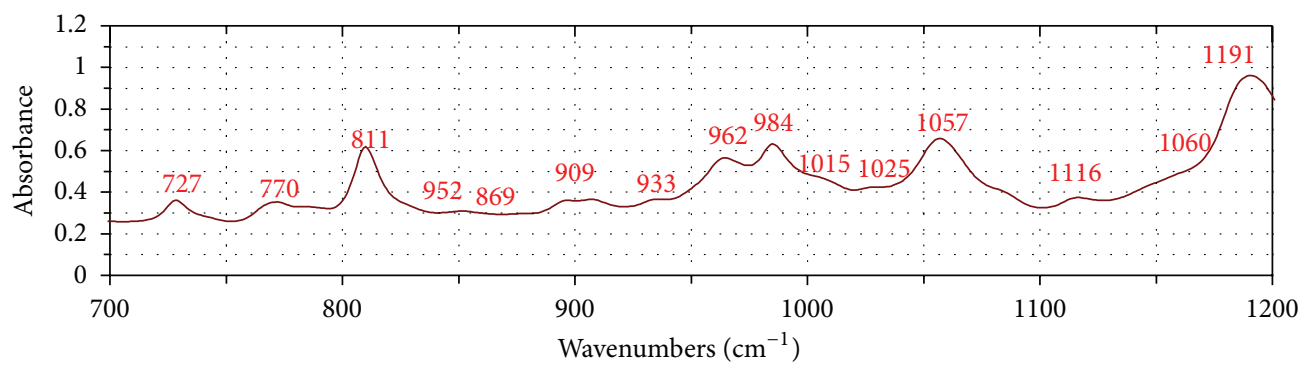

(a)

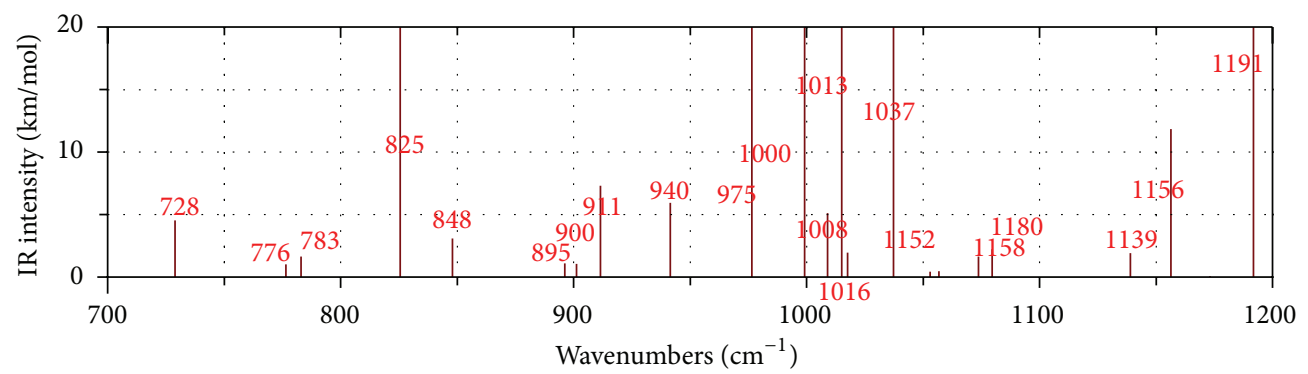

(b)

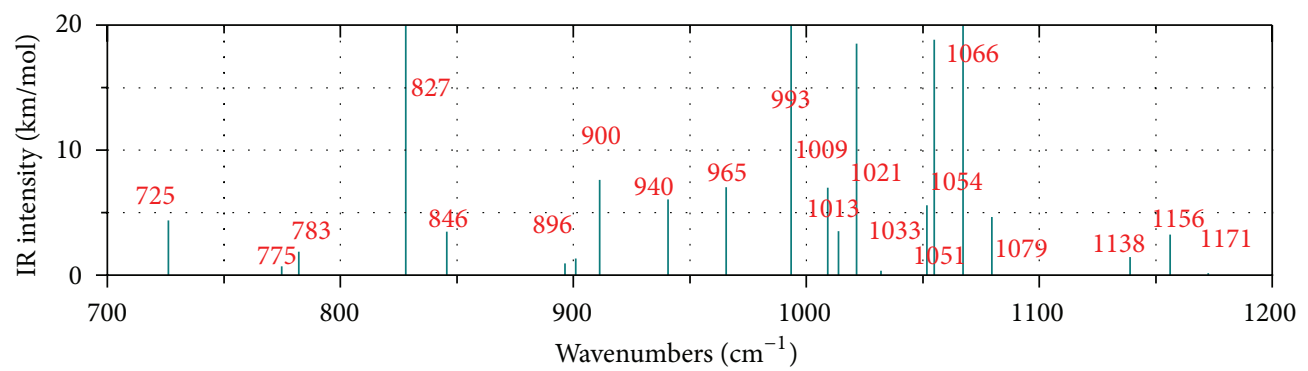

(c)

FIGURE 4: Comparison of the experimental IR (a) CO stretching and $\mathrm{CH}$ bending region $\left(1200-700 \mathrm{~cm}^{-1}\right)$ with the appropriate theoretical $\mathrm{B} 3 \mathrm{LYP} / 6-311+\mathrm{G}^{* *}$ spectra ((b) and (c)) for s-cis and s-trans 2-ethylhexyl acrylate, respectively.

conformation of the title molecule. All the vibrational normal modes in this region are not pure and the majority of them were ascribed according to PED calculations to $\mathrm{CH}$ bending (for more details see Tables 2 and 3 ).

5.4. Region below $700 \mathrm{~cm}^{-1}$. This spectral region includes the bands associated with $\mathrm{C}=\mathrm{C}-\mathrm{C}, \mathrm{C}-\mathrm{O}-\mathrm{C}, \mathrm{O}=\mathrm{C}-\mathrm{O}, \mathrm{O}-\mathrm{C}-$ $\mathrm{C}, \mathrm{C}-\mathrm{C}-\mathrm{C}$, and $\mathrm{C}-\mathrm{C}-\mathrm{C}$ bending and torsion modes about the single bonds $\mathrm{O}-\mathrm{C}$ and $\mathrm{C}-\mathrm{C}$. The frequencies and $\mathrm{PED}$ calculations are presented in Tables 2 and 3. Comparing the 23 first normal modes for the two conformers, we notice that they have the same type of vibrational modes. However, the normal modes 16,17 , and 18 represent CCC bending in the strans and $\mathrm{CH}$ and $\mathrm{CCC}$ rocking modes in the s-cis conformer.

Due to the lack of experimental data on this region, we compare our calculated frequencies to some experimental IR bands of related molecules presented in the literature. The bands at $356,254,116$, and $100 \mathrm{~cm}^{-1}$ are close to the bands at $349\left(\mathrm{~A}^{\prime}\right), 244\left(\mathrm{~A}^{\prime \prime}\right), 114\left(\mathrm{~A}^{\prime \prime}\right)$, and $114 \mathrm{~cm}^{-1}\left(\mathrm{~A}^{\prime \prime}\right)$ reported by Dulce et al. [28]. From the same work the bands at 530, 244, 114 , and $114 \mathrm{~cm}^{-1}$ belonging to $A^{\prime \prime}$ symmetry, for the s-trans methyl acrylate in liquid state, are close to our calculated bands in DFT at 542, 244, 116, and $106 \mathrm{~cm}^{-1}$.

\section{Conclusions}

The mid-IR spectrum of 2-ethylhexyl acrylate was measured and interpreted with support of the DFT//B3LYP/6-311+G ${ }^{* *}$ calculated vibrational spectra followed by potential energy distribution analysis. Assuming the s-cis and s-trans conformations, we found two stable conformations, close in their energies. The fully optimized geometries of s-cis and s-trans conformations by DFT//B3LYP/6-311+G ${ }^{* *}$ were compared with the experimental and theoretical data presented in the literature on the methyl acrylate molecule. The comparison shows a good agreement. The experimental vibrational spectrum is in good agreement with the theoretical spectra calculated for the two conformations. Two neighbor bands in the IR spectrum at 1619 and $1637 \mathrm{~cm}^{-1}$ may be used as characteristic bands to locate and distinguish the existence of one or both conformations. 


\section{References}

[1] M. Salkind, E. H. Riddle, and R. W. Keefer, "Acrylates and methacrylates: ester manufacture and markets," Industrial \& Engineering Chemistry Research, vol. 51, no. 11, pp. 1328-1334, 1959.

[2] K. S. Anseth, S. M. Newman, and C. N. Bowman, "Polymeric dental composites: properties and reaction behavior of multimethacrylate dental restorations," Advances in Polymer Science, vol. 122, pp. 176-217, 1995.

[3] J. G. Kloosterboer, "Network formation by chain crosslinking photopolymerization and its application in electronics," Advances in Polymer Science, vol. 84, pp. 1-61, 1988.

[4] K. Matyjaszewski, Y. Gnanou, and L. Leibler, Macromolecular Engineering, vol. 1, Wiley-VCH, Weinheim, Germany, 2007.

[5] M. Orgill, B. L. Baker, and N. L. Owen, "FTIR studies of conformational isomerism in acrylates and acrylic acids," Spectrochimica Acta A, vol. 55, no. 5, pp. 1021-1024, 1999.

[6] M. Salkind, "Reaches into the market," Industrial \& Engineering Chemistry Research, vol. 56, pp. 62-110, 1964.

[7] E. Takács, K. Dajka, and L. Wojnárovits, "Study of high-energy radiation initiated polymerization of butyl acrylate," Radiation Physics and Chemistry, vol. 63, no. 1, pp. 41-44, 2002.

[8] H. Yu, J. Peng, M. Zhai, J. Li, G. Wei, and J. Qiao, "Radiationinduced copolymerization of styrene/n-butyl acrylate in the presence of ultra-fine powdered styrene-butadiene rubber," Radiation Physics and Chemistry, vol. 76, no. 11-12, pp. 17361740, 2007.

[9] P. F. Cañamero, J. Luis de la Fuente, and M. Fermández-García, "Curing kinetic study using a well-controlled multifunctional copolymer based on glycidyl methacrylate," European Polymer Journal, vol. 45, no. 9, pp. 2665-2673, 2009.

[10] D. Mathew, C. P. Reghunadhan Nair, and K. N. Ninan, "Pendant cyanate functional vinyl polymers and imidophenolic-triazines thereof: synthesis and thermal properties," European Polymer Journal, vol. 36, no. 6, pp. 1195-1208, 2000.

[11] S. Mitra, S. Chattopadhyay, S. Sabharwal, and A. K. Bhowmick, "Electron beam crosslinked gels-Preparation, characterization and their effect on the mechanical, dynamic mechanical and rheological properties of rubbers," Radiation Physics and Chemistry, vol. 79, no. 2, pp. 289-296, 2010.

[12] P. Cañamero-Martínez, M. Fermández-García, and J. Luis de la Fuente, "Rheological cure characterization of a polyfunctional epoxy acrylic resin," Reactive and Functional Polymers, vol. 70, no. 10, pp. 761-766, 2010.

[13] F. Chu, T. McKenna, and S. Lu, "Curing kinetics of an acrylic resin/epoxy resin system using dynamic scanning calorimetry," European Polymer Journal, vol. 33, no. 6, pp. 837-840, 1997.

[14] M. C. Douskey, M. S. Gebhard, A. V. McCormick et al., "Spectroscopic studies of a novel cyclic oligomer with pendant alkoxysilane groups," Progress in Organic Coatings, vol. 45, no. 2-3, pp. 145-157, 2002.

[15] R. J. Day, P. A. Lovell, and A. A. Wazzan, "Toughened carbon/epoxy composites made by using core/shell particles," Composites Science and Technology, vol. 61, no. 1, pp. 41-56, 2001.

[16] K. Bolton, D. G. Lister, and J. Sheridan, "Rotational isomerism, barrier to internal rotation and electric dipole moment of acrylic acid by microwave spectroscopy," Journal of the Chemical Society, Faraday Transactions 2, vol. 70, pp. 113-123, 1974.

[17] S. W. Charles, F. C. Cullen, N. L. Owen, and G. A. Williams, "Infrared spectrum and rotational isomerism of acrylic acid," Journal of Molecular Structure, vol. 157, no. 1-3, pp. 17-25, 1987.
[18] K. Bolton, N. L. Owen, and J. Sheridan, "Microwave spectra of rotational isomers of acrylic acid," Nature, vol. 218, no. 5138, pp. 266-267, 1968.

[19] G. Williams, N. L. Owen, and J. Sheridan, "Spectroscopic studies of some substituted methyl formates-part 1: microwave spectra and internal rotation barriers of methyl-fluoroformate, -propiolate, -cyanoformate, -acrylate and -acetate," Transactions of the Faraday Society, vol. 67, pp. 922-949, 1971.

[20] T. Tsuji, H. Ito, H. Takeuchi, and S. Konaka, "Molecular structure and conformation of methyl methacrylate determined by gas electron diffraction," Journal of Molecular Structure, vol. 475, no. 1, pp. 55-63, 1999.

[21] T. Egawa, S. Maekawa, H. Fujiwara Takeuchi, H. Takeuchi, and S. Konaka, "Molecular structure and conformation of methyl acrylate: a gas electron diffraction study augmented by ab initio calculation and rotational constants," Molecular Structure and Spectroscopy, vol. 352, pp. 193-201, 1995.

[22] A. Virdi, V. P. Gupta, and A. Sharma, "Ab initio studies on conformation, vibrational and electronic spectra of methyl methacrylate," Journal of Molecular Structure, vol. 634, no. 5, pp. 53-65, 2003.

[23] B. L. Baker, M. Orgill, N. L. Owen et al., "The molecular conformation of methyl methacrylate-an infrared and ab initio study," Journal of Molecular Structure, vol. 356, no. 2, pp. 95-104, 1995.

[24] A. N. Mitra and I. Santhanan, "Relativistic qqq spectra from Bethe-Salpeter premises," Physics Letters B, vol. 104, no. 1, pp. 62-66, 1981.

[25] K. Fan and J. E. Boggs, "Rotational isomerism of acrylic acid," Journal of Molecular Structure, vol. 157, no. 1-3, pp. 31-41, 1987.

[26] P. Carmona and J. Moreno, "The infrared spectra and structure of methyl acrylate," Journal of Molecular Structure, vol. 82, no. 3-4, pp. 177-185, 1982.

[27] R. J. Loncharich, T. R. Schwartz, and K. N. Houk, “Theoretical studies of conformations of acrolein, acrylic acid, methyl acrylate, and their Lewis acid complexes," Journal of the American Chemical Society, vol. 109, no. 1, pp. 14-23, 1987.

[28] M. Dulce, G. Faria, J. J. C. Teixeira-Dias, and R. Fausto, "Vibrational spectra and structure of methyl trans-crotonate," Vibrational Spectroscopy, vol. 2, no. 2-3, pp. 43-60, 1991.

[29] J. J. C. Teixeira-Dias and R. Fausto, "Molecular structure of methyl acrylate: the high energy s-trans-(CO) conformer," Journal of Molecular Structure, vol. 282, no. 1-2, pp. 123-129, 1993.

[30] A. J. Bowles, W. O. George, and D. B. Cunliffe-Jones, "Conformations of some $\alpha \beta$-unsaturated carbonyl compounds-part II: infrared and Raman spectra of methyl and ethyl acrylates and transcrotonates," Journal of the Chemical Society B, pp. 10701075, 1970.

[31] A. D. Becke, "Density-functional thermochemistry. III. The role of exact exchange," The Journal of Chemical Physics, vol. 98, no. 7, pp. 5648-5652, 1993.

[32] C. Lee, W. Yang, and R. G. Parr, "Development of the ColleSalvetti correlation-energy formula into a functional of the electron density," Physical Review B, vol. 37, no. 2, pp. 785-789, 1988.

[33] H. Yoshida, K. Takeda, J. Okamura, A. Ehara, and H. Matsuura, "A new approach to vibrational analysis of large molecules by density functional theory: wavenumber-linear scaling method," Journal of Physical Chemistry A, vol. 106, no. 14, pp. 3580-3586, 2002. 
[34] H. Yoshida, A. Ehara, and H. Matsuura, "Density functional vibrational analysis using wavenumber-linear scale factors," Chemical Physics Letters, vol. 325, no. 4, pp. 477-483, 2000.

[35] M. J. Frisch, G. W. Trucks, H. B. Schlegel et al., Gaussian 03, Revision B. 01, Gaussian, Pittsburgh, Pa, USA, 2003.

[36] J. M. L. Martin and C. Van Alsenoy, GAR2PED, A Program to Obtain a Potential Energy Distribution from a Gaussian Archive Record, University of Antwerp, 2009.

[37] P. Pulay, G. Fogarasi, F. Pang, and J. E. Boggs, "Systematic ab initio gradient calculation of molecular geometries, force constants, and dipole moment derivatives," Journal of the American Chemical Society, vol. 101, no. 10, pp. 2550-2560, 1979.

[38] S. Mishra, D. Chaturvedi, N. Kumar, P. Tandon, and H. W. Siesler, "An ab initio and DFT study of structure and vibrational spectra of $\gamma$ form of Oleic acid: comparison to experimental data," Chemistry and Physics of Lipids, vol. 163, no. 2, pp. 207217, 2010.

[39] W. O. George, D. V. Hassid, and W. F. Maddams, "Conformations of some $\alpha \beta$-unsaturated carbonyl compounds-part III: infrared solution spectra of methyl, [2H3]methyl, ethyl, and [2H5] ethyl acrylates and trans-crotonates," Journal of the Chemical Society, Perkin Transactions 2, no. 4, pp. 400-404, 1972. 

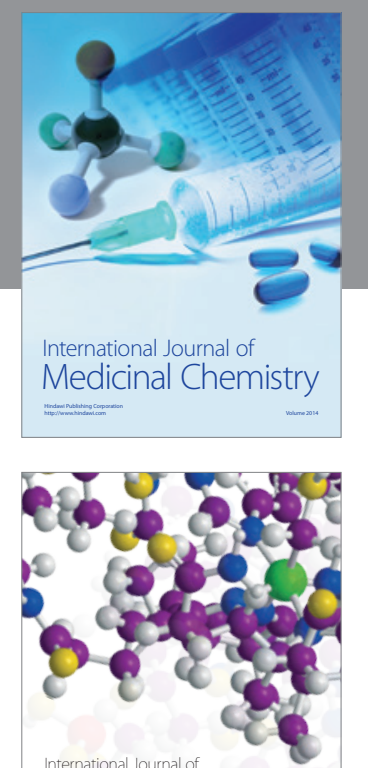

\section{Carbohydrate} Chemistry

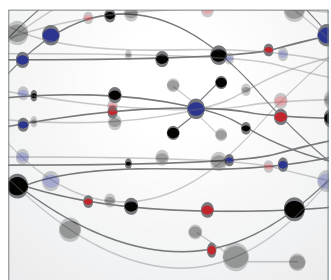

The Scientific World Journal
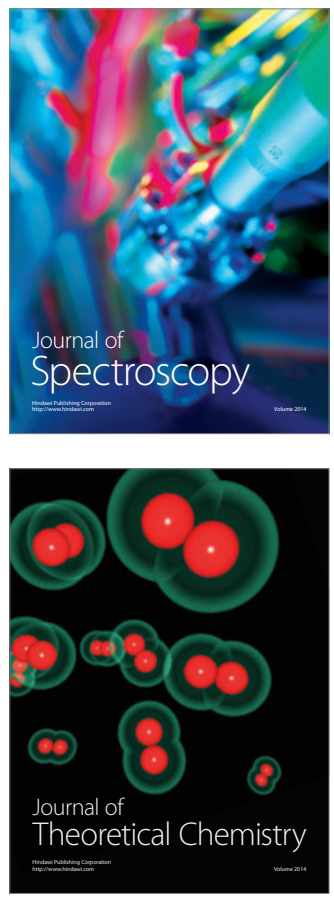
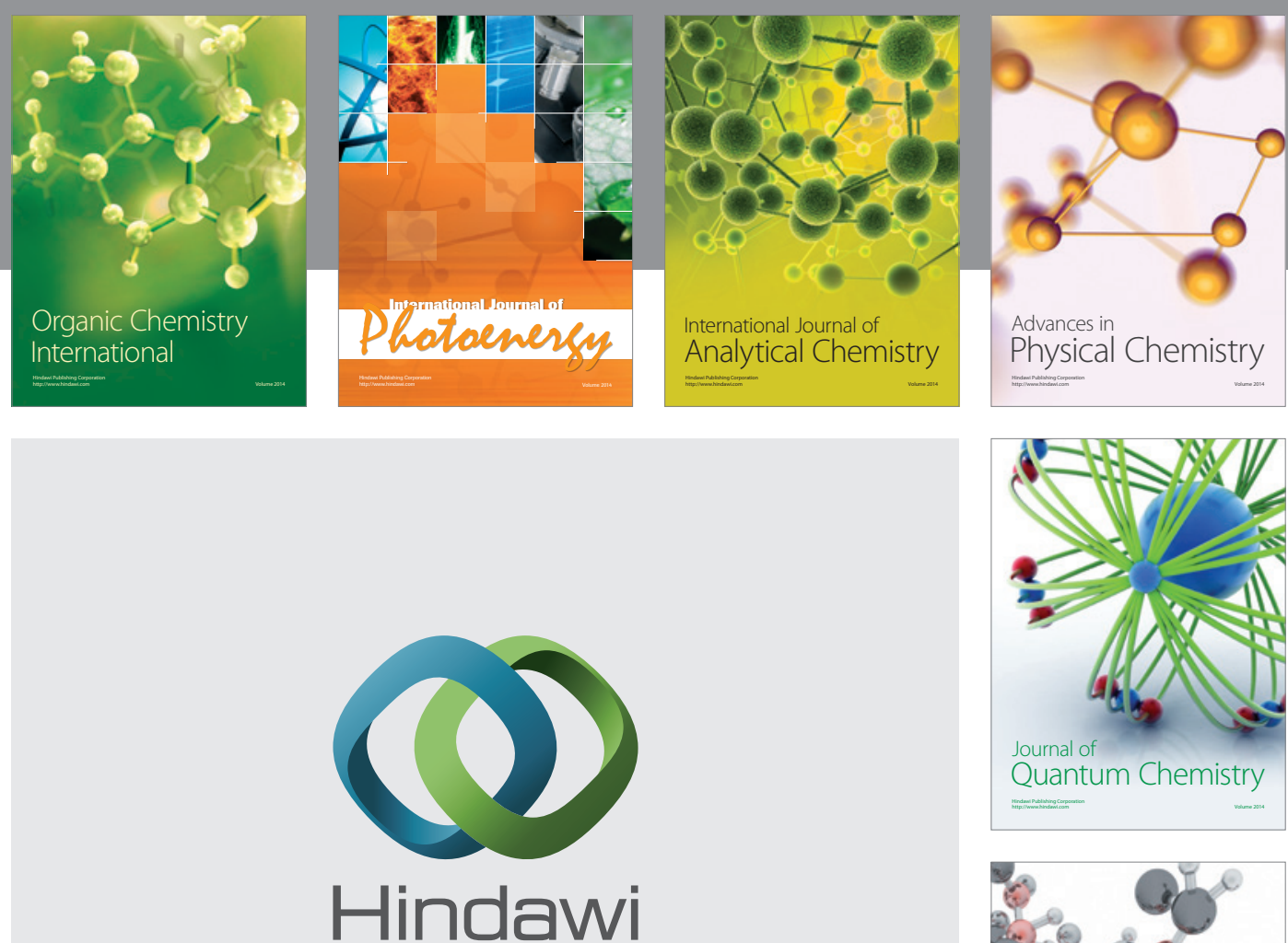

Submit your manuscripts at

http://www.hindawi.com

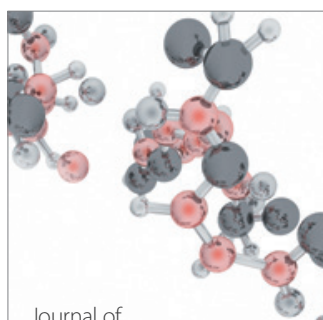

Analytical Methods

in Chemistry

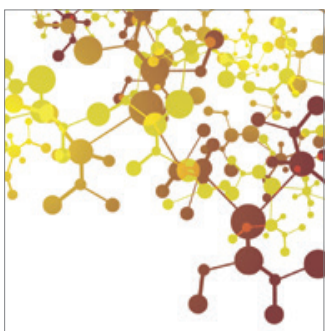

Journal of

Applied Chemistry

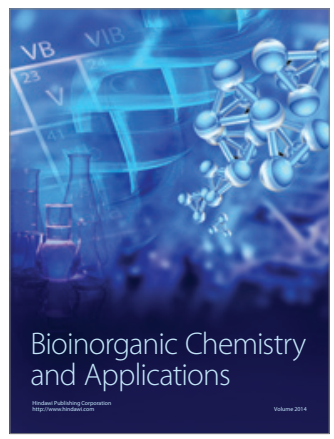

Inorganic Chemistry
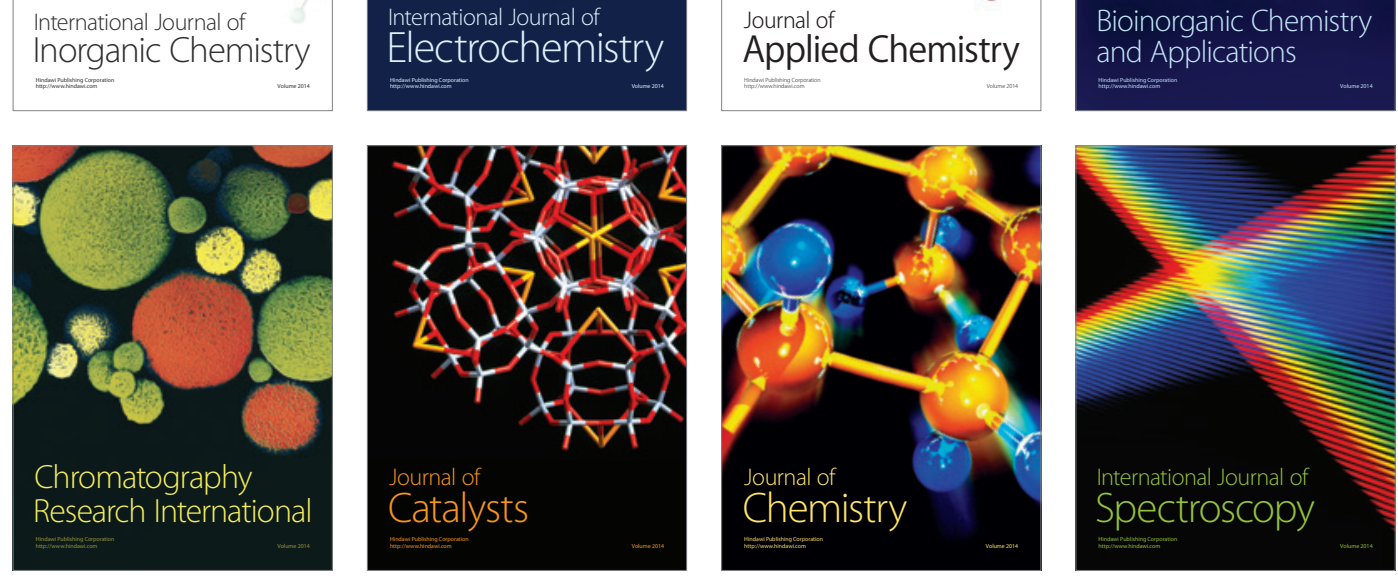